\title{
Boronate titanium alkylidene reagents for the diversity-based synthesis of benzofurans
}

\author{
Gordon J. McKiernan and Richard C. Hartley \\ Department of Chemistry, University of Glasgow, Glasgow, G12 8QQ, Scotland \\ richh@chem.gla.ac.uk
}

\section{Supporting Information}

General considerations: All experiments were performed under an atmosphere of $\mathrm{Ar}$ or $\mathrm{N}_{2}$. THF was distilled from sodium/benzophenone; $\mathrm{P}(\mathrm{OEt})_{3}$ was distilled from $\mathrm{CaH}_{2}$ prior to use and $\mathrm{DMF}$ was distilled from $\mathrm{CaH}_{2}$ then stored over activated $4 \AA \mathrm{MS}$. Bis(cyclopentadienyl)titanium dichloride was purchased from Lancaster and used without further purification. Merrifield resin was purchased from Polymer laboratories and had a loading of $1.83 \mathrm{mmol} / \mathrm{g}$. Resin-bound esters were synthesised according to the procedure of Frenette and Friesen. ${ }^{1}$

Example procedure: Bis(cyclopentadienyl)titanium dichloride (0.93g , $3.74 \mathrm{mmol}, 12 \mathrm{eq})$, magnesium turnings $(0.10 \mathrm{~g}, 4.12 \mathrm{mmol}, 13.2 \mathrm{eq})$ and $4 \AA \mathrm{MS}(0.20 \mathrm{~g})$ were heated gently by heat-gun for $1 \mathrm{~min}$, under reduced pressure. Once cooled, THF $(4 \mathrm{ml})$ and $\mathrm{P}(\mathrm{OEt})_{3}(1.30 \mathrm{ml}, 8.07 \mathrm{mmol}, 24 \mathrm{eq})$ were added and the mixture stirred at room temperature. After $3 \mathrm{~h}$, a solution of [1,3]dithiane $23(0.38 \mathrm{~g}, 0.933$ mmol, $3 \mathrm{eq})$ in THF $(10 \mathrm{ml})$ was added and the mixture stirred for a further $15 \mathrm{~min}$ before addition of Merrifield resin-bound ester $\mathbf{1 2 b}(0.311 \mathrm{meq}$, contained within an IRORI macrokan). The mixture was heated to reflux. After $3 \mathrm{~h}$, the reaction was allowed to cool, the resin removed and washed: THF (5 x $30 \mathrm{ml}$ ); $\mathrm{MeOH}$ and DCM alternately (4 x $30 \mathrm{ml}$ each); and finally $\mathrm{MeOH}$ and DCM (30ml each). The resin-bound enol ether was dried in vacuo.

The IRORI macrokan containing resin-bound enol ether $\mathbf{3 1 b}(0.311 \mathrm{meq})$ was treated with $\mathrm{Cs}_{2} \mathrm{CO}_{3}(0.52 \mathrm{~g}, 1.56 \mathrm{mmol}, 5 \mathrm{eq}), \mathrm{Pd}\left(\mathrm{PPh}_{3}\right)_{4}(14.7 \mathrm{mg}, 4 \mathrm{~mol} \%)$ and iodotoluene $(0.33 \mathrm{~g}, 1.56 \mathrm{mmol}, 5$ eq) in dry DMF $(8 \mathrm{ml})$ and distilled water $(5.6 \mu \mathrm{l}, 0.311 \mathrm{mmol}, 1 \mathrm{eq})$ was added. The mixture was heated to $80{ }^{\circ} \mathrm{C}$. After $20 \mathrm{~h}$, the reaction was cooled and the resin washed: (9:1) DMF- $\mathrm{H}_{2} \mathrm{O}(3 \times 30 \mathrm{ml})$; THF (5 x $30 \mathrm{ml}) ; \mathrm{MeOH}$ and DCM alternately (4 x $30 \mathrm{ml}$ each); and finally, $\mathrm{MeOH}$ and DCM (30 ml each). The resin was dried in vacuo then treated with 1\%TFA-DCM for $30 \mathrm{~min}$. The DCM solution was decanted then the resin washed with DCM (3 x $5 \mathrm{ml})$; all DCM solutions were combined, neutralised/dried over solid anhydrous $\mathrm{K}_{2} \mathrm{CO}_{3}$ and concentrated in vacuo to give the ketone 32ba' as an orange oil (57 mg, $0.177 \mathrm{mmol}, 57 \%$ based on loading of chloromethylpolystyrene). The orange oil (54 $\mathrm{mg}, 0.166 \mathrm{mmol}$ after characterisation) was taken-up in $10 \%$ conc. $\mathrm{HCl}-\mathrm{MeOH}(5 \mathrm{ml})$ and heated to reflux for $5 \mathrm{~min}$. $\mathrm{MeOH}$ was removed in vacuo and the residue taken-up in DCM (50 ml) and dried/neutralised over solid $\mathrm{K}_{2} \mathrm{CO}_{3}$ then concentrated in vacuo to give the benzo/b]furan 33ba'as a yellow solid (41.5 mg, $0.158 \mathrm{mmol}, 95 \%)$.

${ }^{1}$ 1. Frenette, R.; Friesen, R.W.; Tetrahedron Lett. 1994, 35, 9177 
Crystal stucture of (5'-Bromophenyl-2'-tert-butyldimethylsiloxyphenyl)-[1,3]dithiane 24:

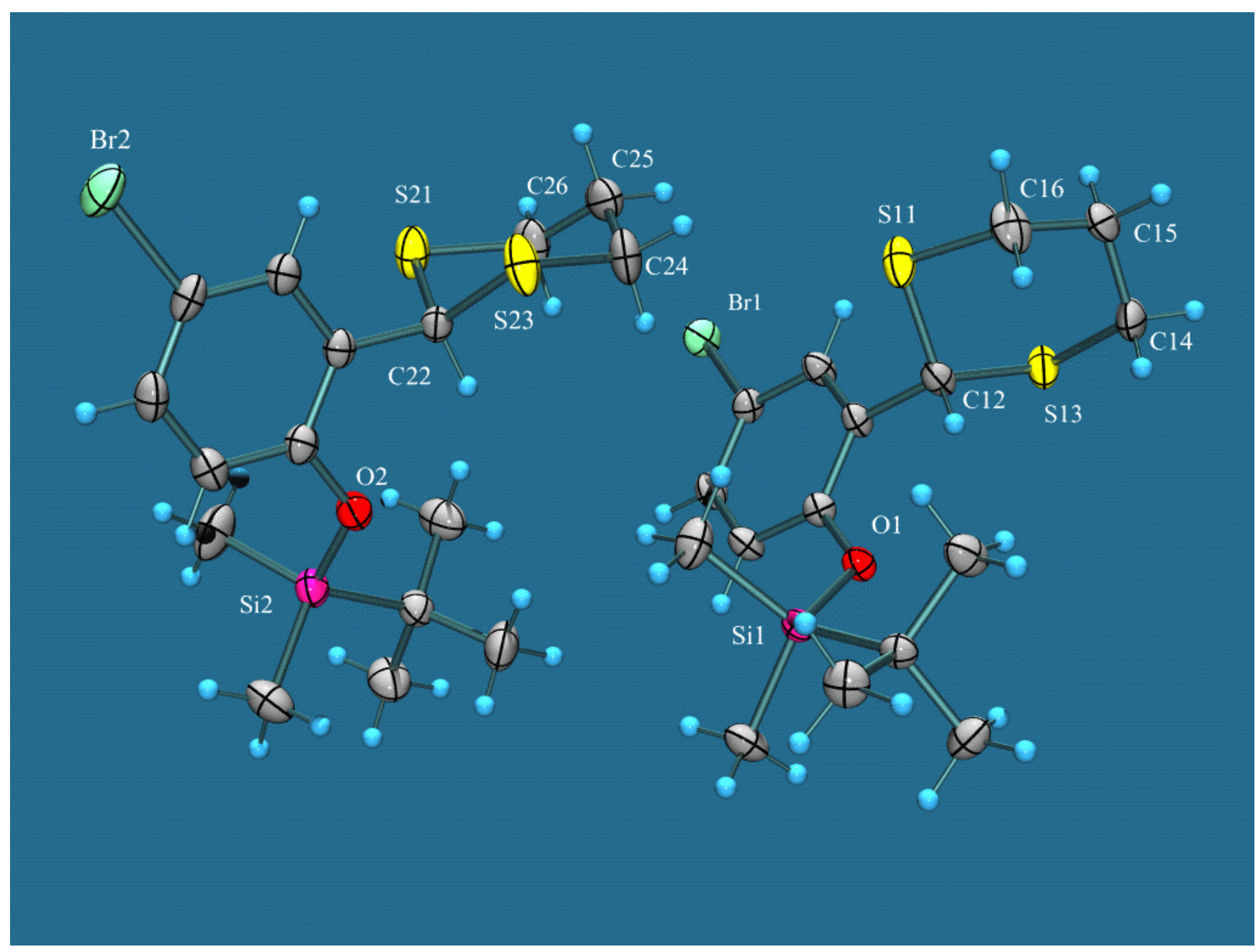




\section{${ }^{1} \mathrm{H}$ NMR spectra of $14-16,28 a-c, 29 a-c$ and $32 a a^{\prime}-c c '$}

1-[4'-(4",4",5",5"-Tetramethyl-1",3",2"-dioxaborolan-2"-yl)phenyl]-4-phenylbutan-2-one 14
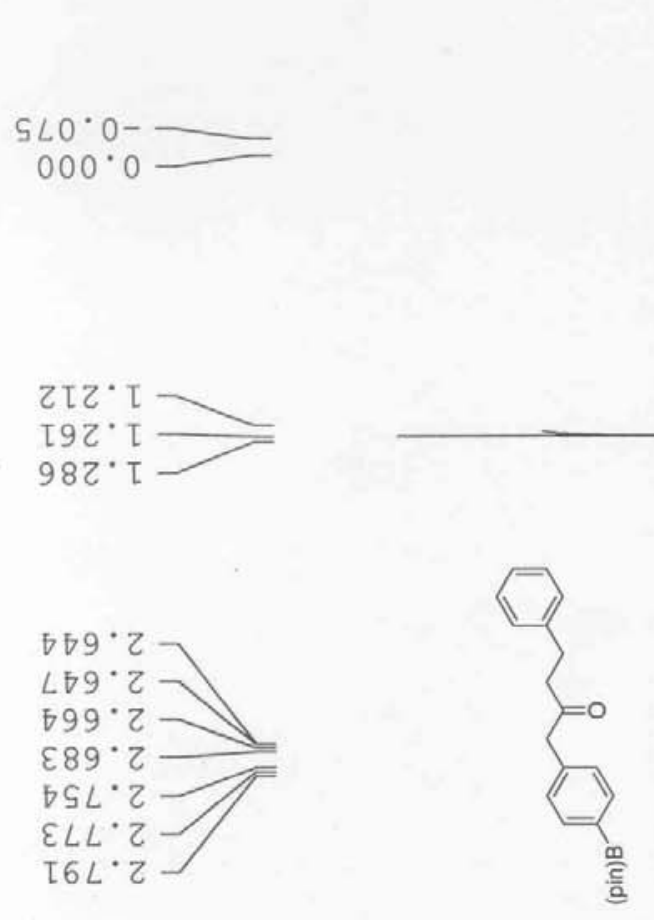

$\tau 6 S^{\cdot} \varepsilon$
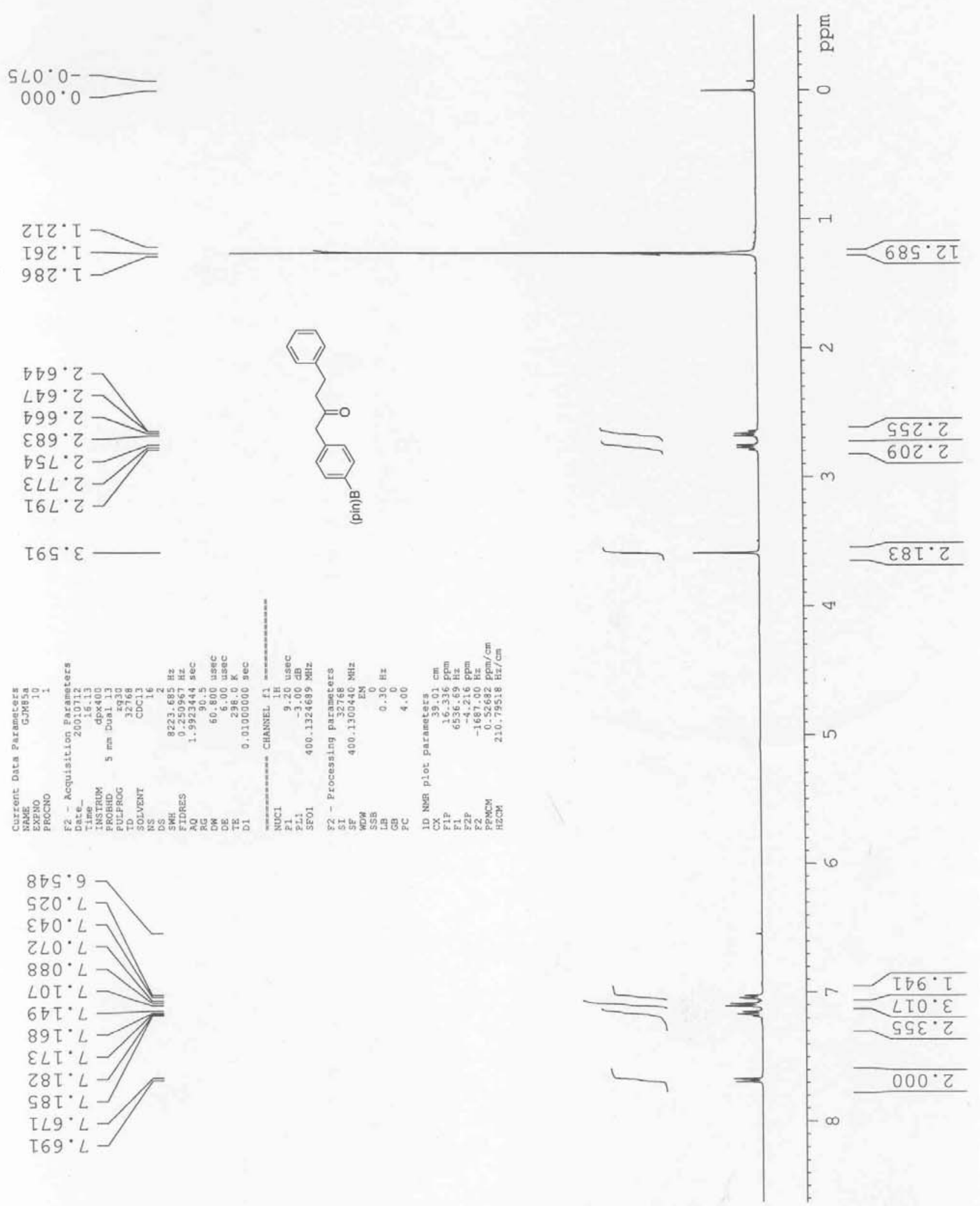
1-(4'-methylphenyl)phenyl-4-phenylbutan-2-one 15
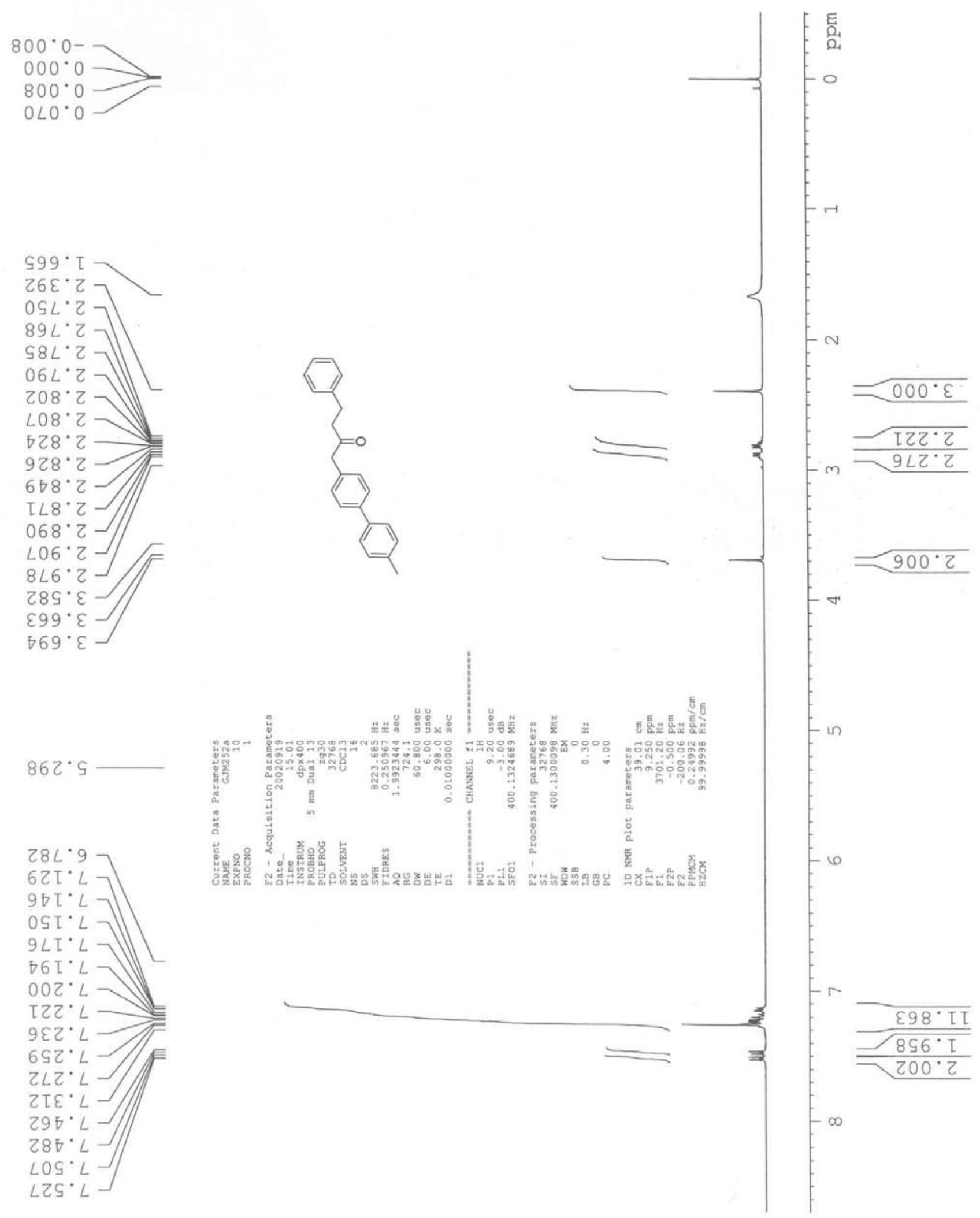

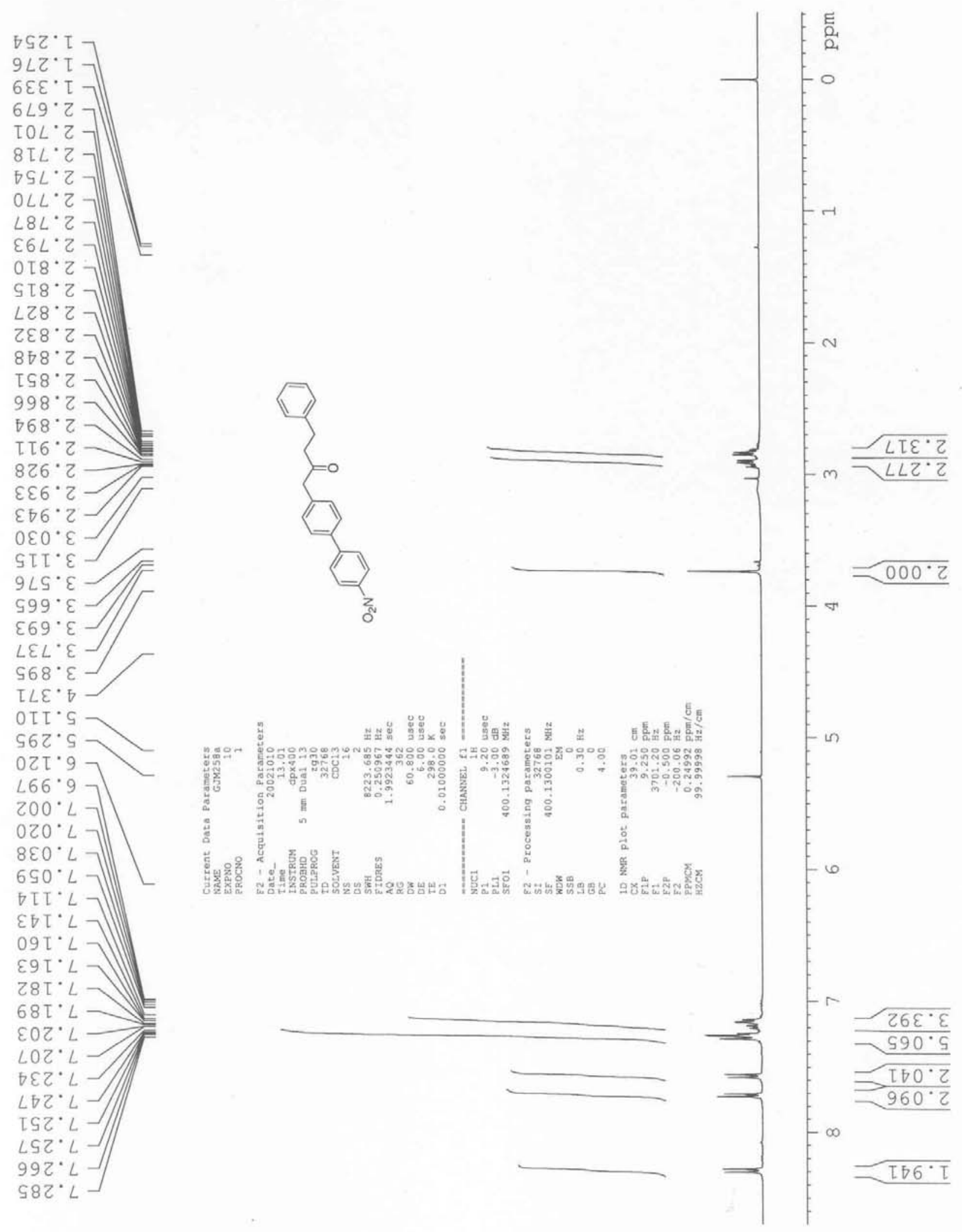
-4-phenylbutan-2-one 28a

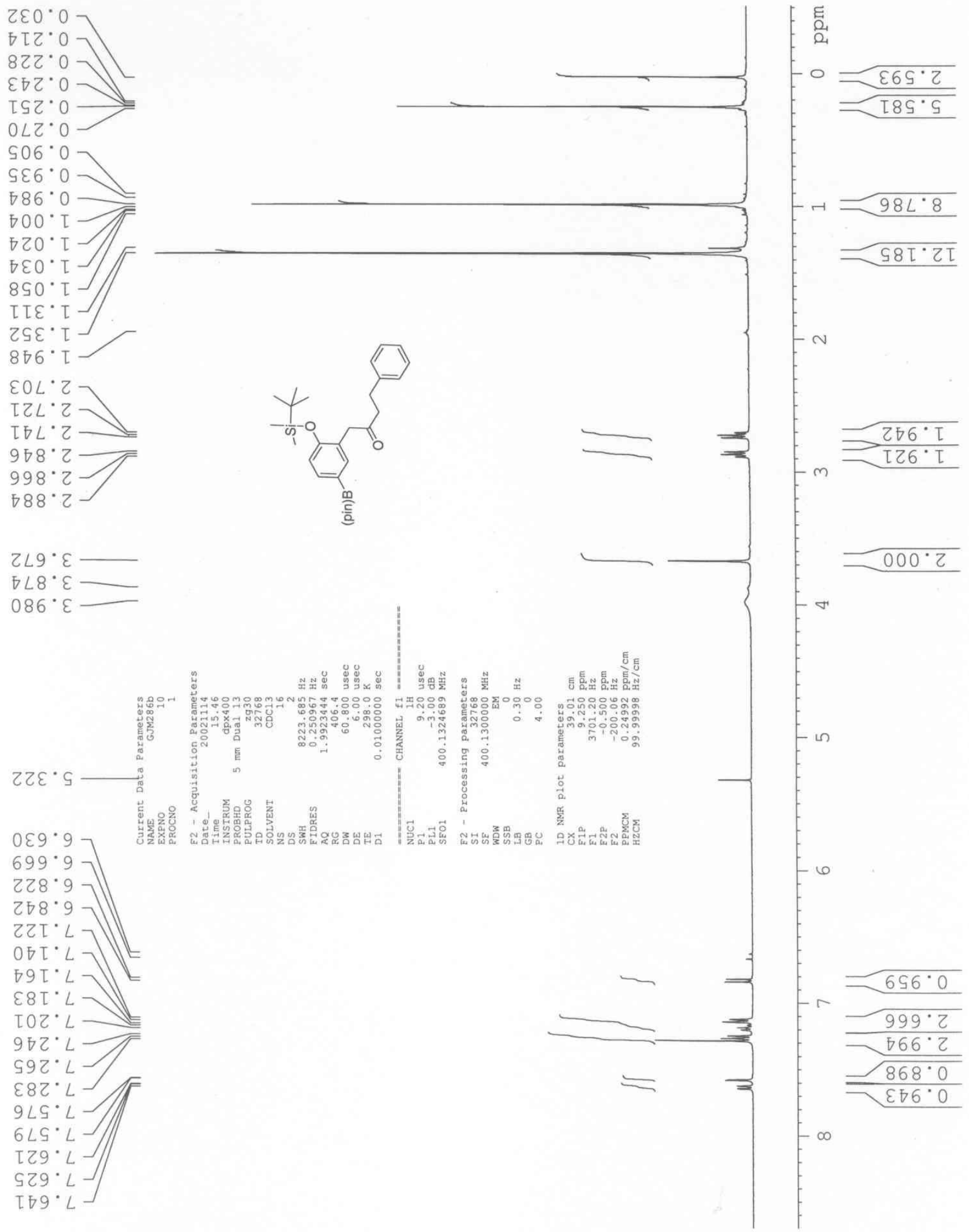


1-[5'-(4",4",5",5"-Tetramethyl-1",3",2"-dioxaborolan-2"'-yl)-2'-tert-butyldimethylsiloxyphenyl] $-3,3$-dimethoxyacrylone $28 b$
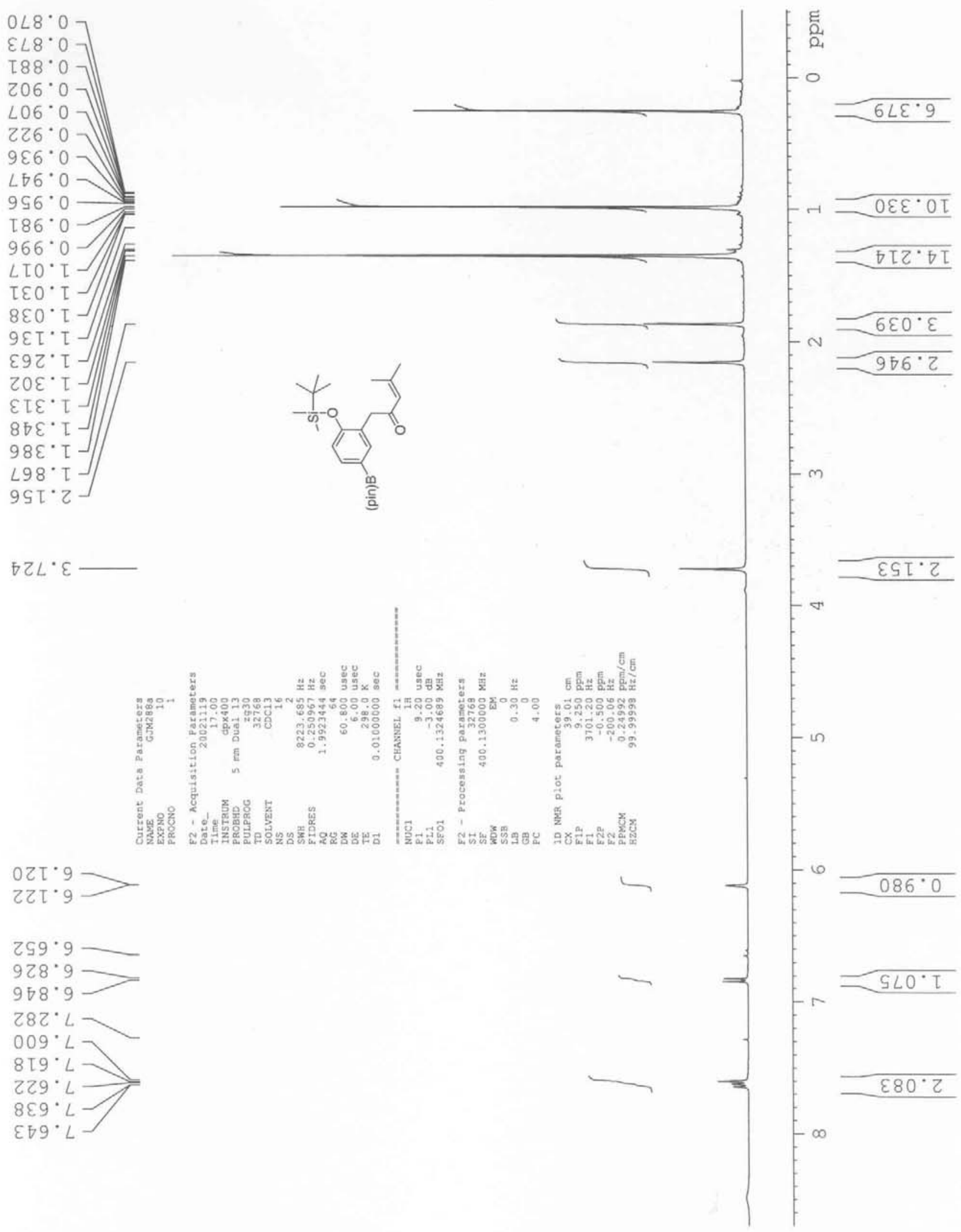
1-[5'-(4",4",5",5"-Tetramethyl-1",3",2"-dioxaborolan-2"'-yl)-2'-tert-butyldimethylsiloxyphenyl] -para-methoxyacetophenone $28 \mathrm{c}$
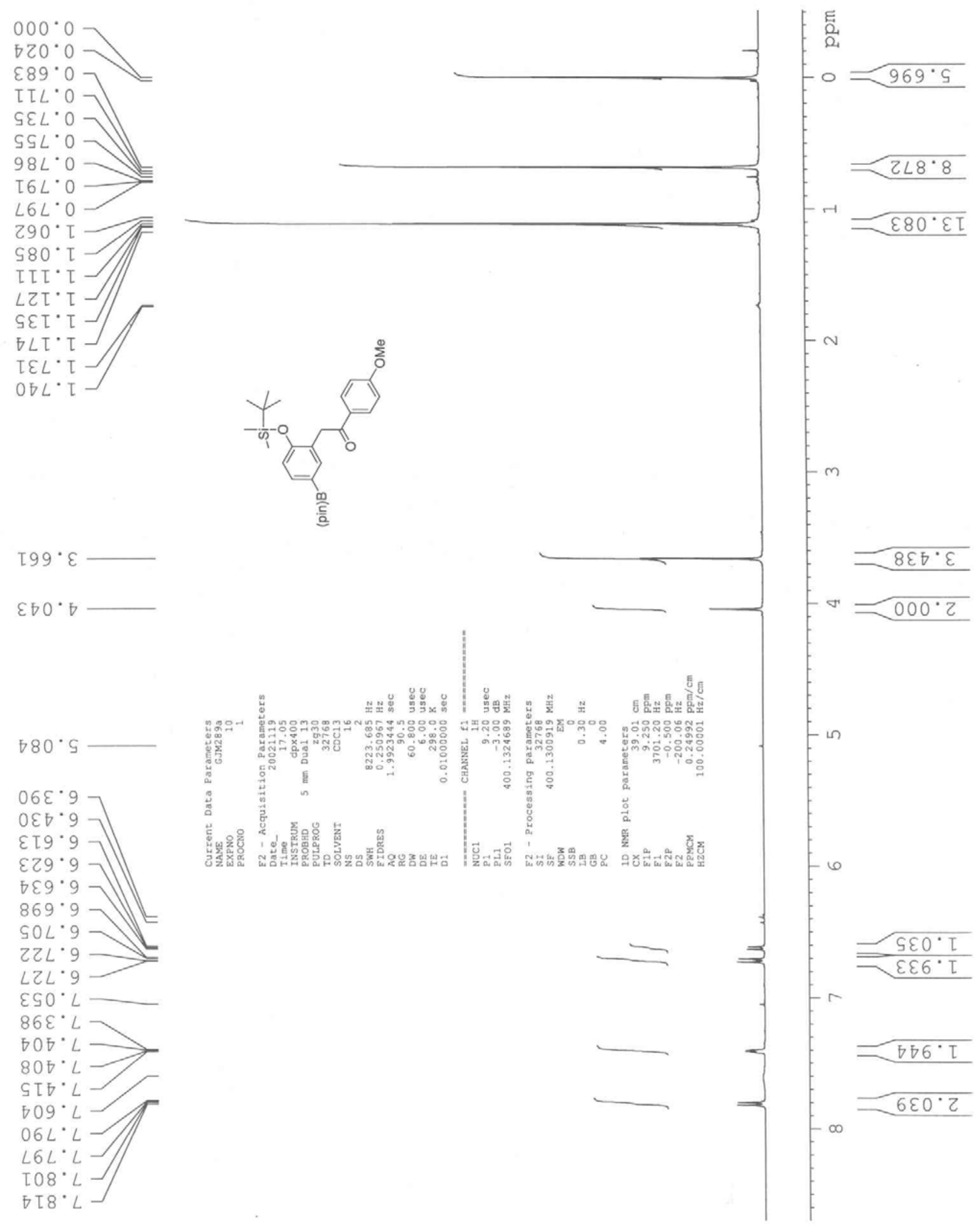


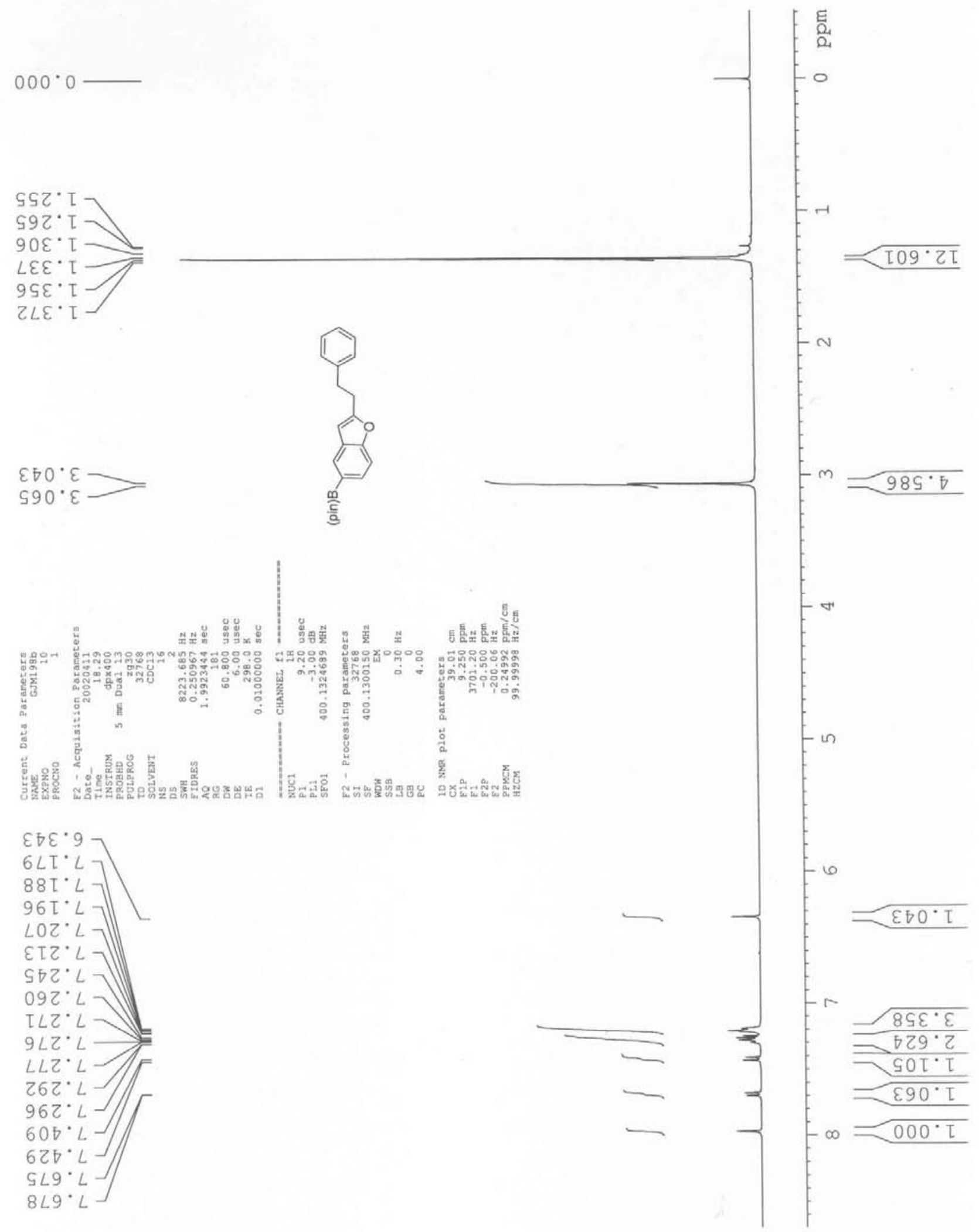




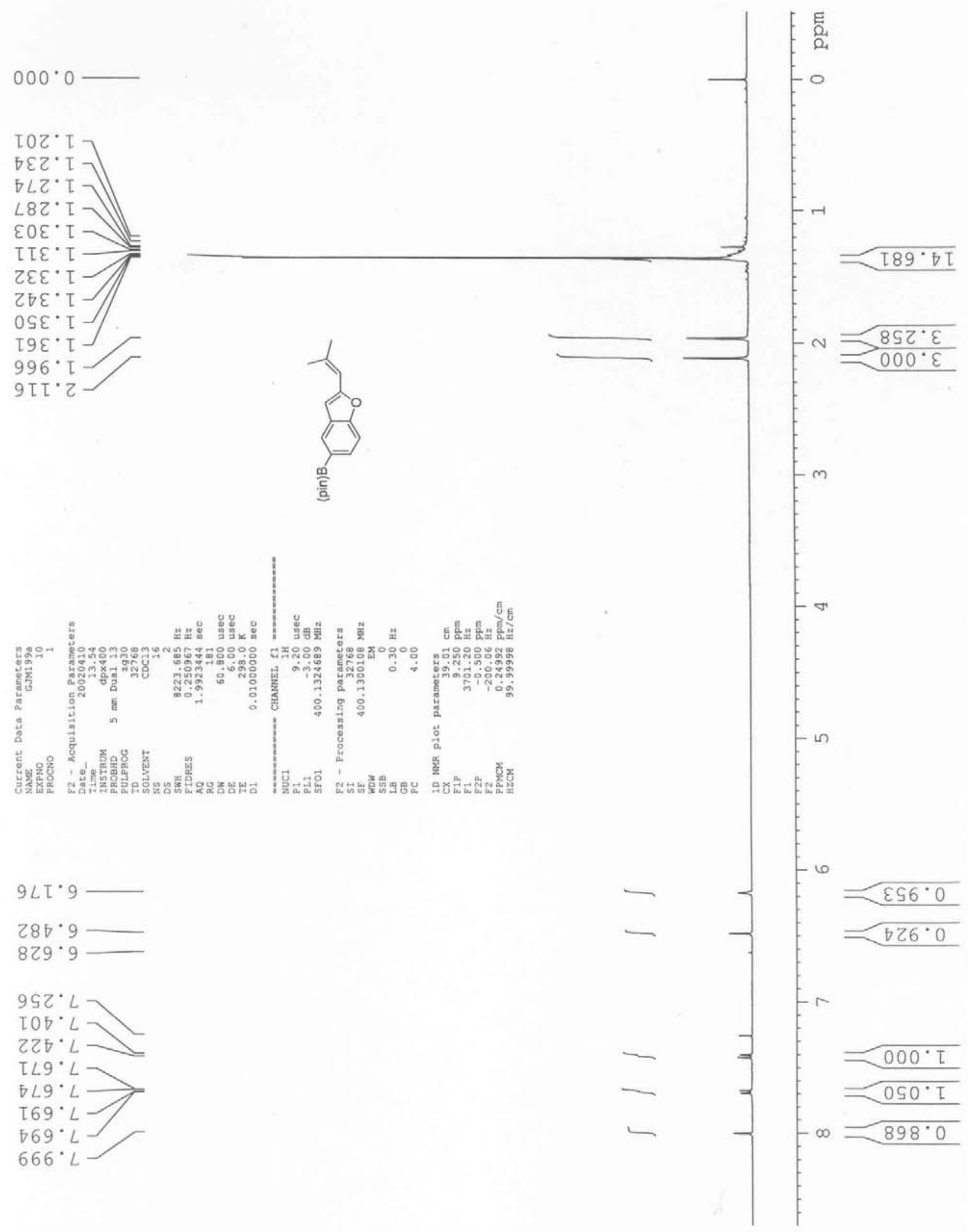



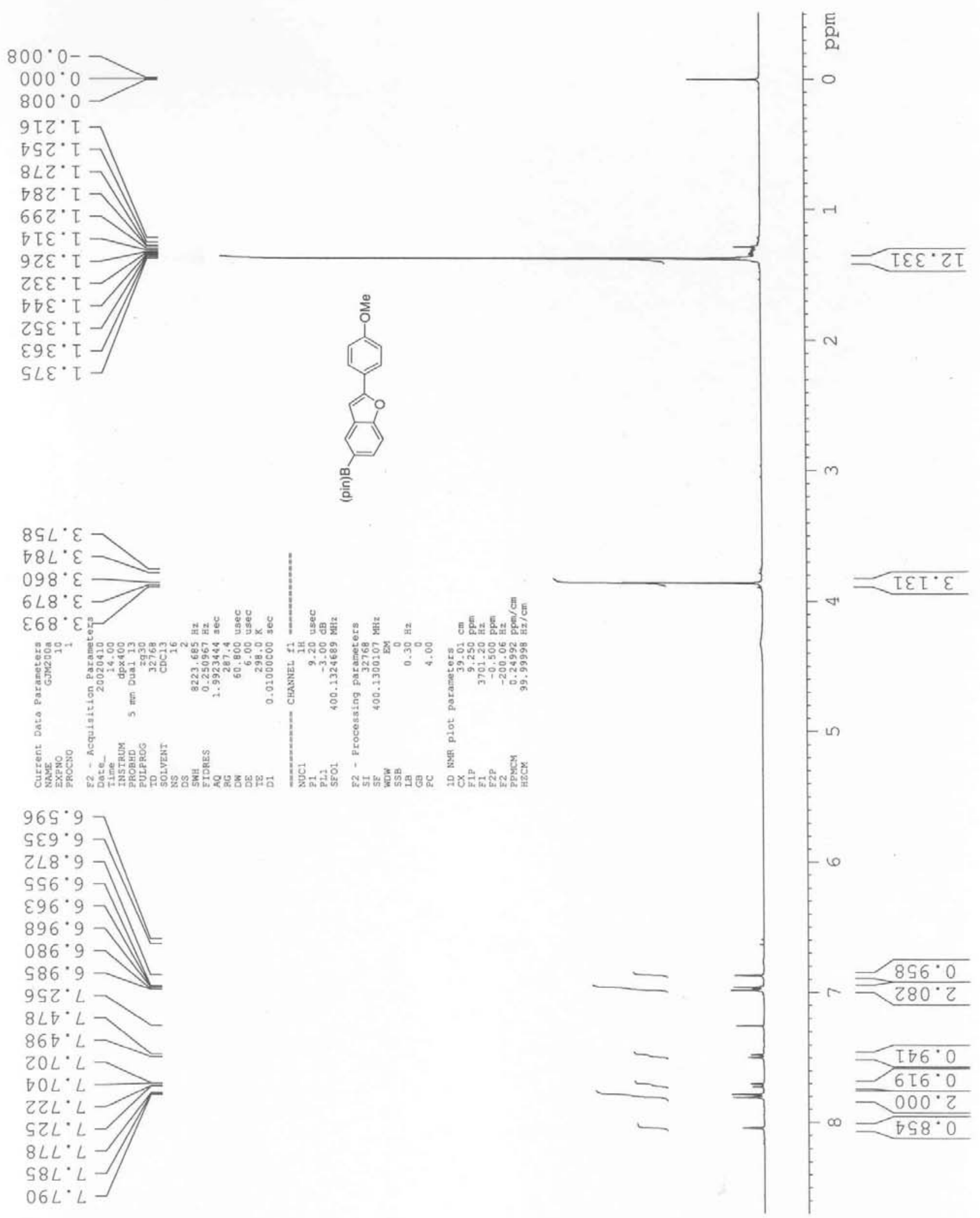
1-[2'-Methoxymethoxy-5'-(4'--methylphenyl)phenyl]-4-phenylbutan-2-one 32aa'

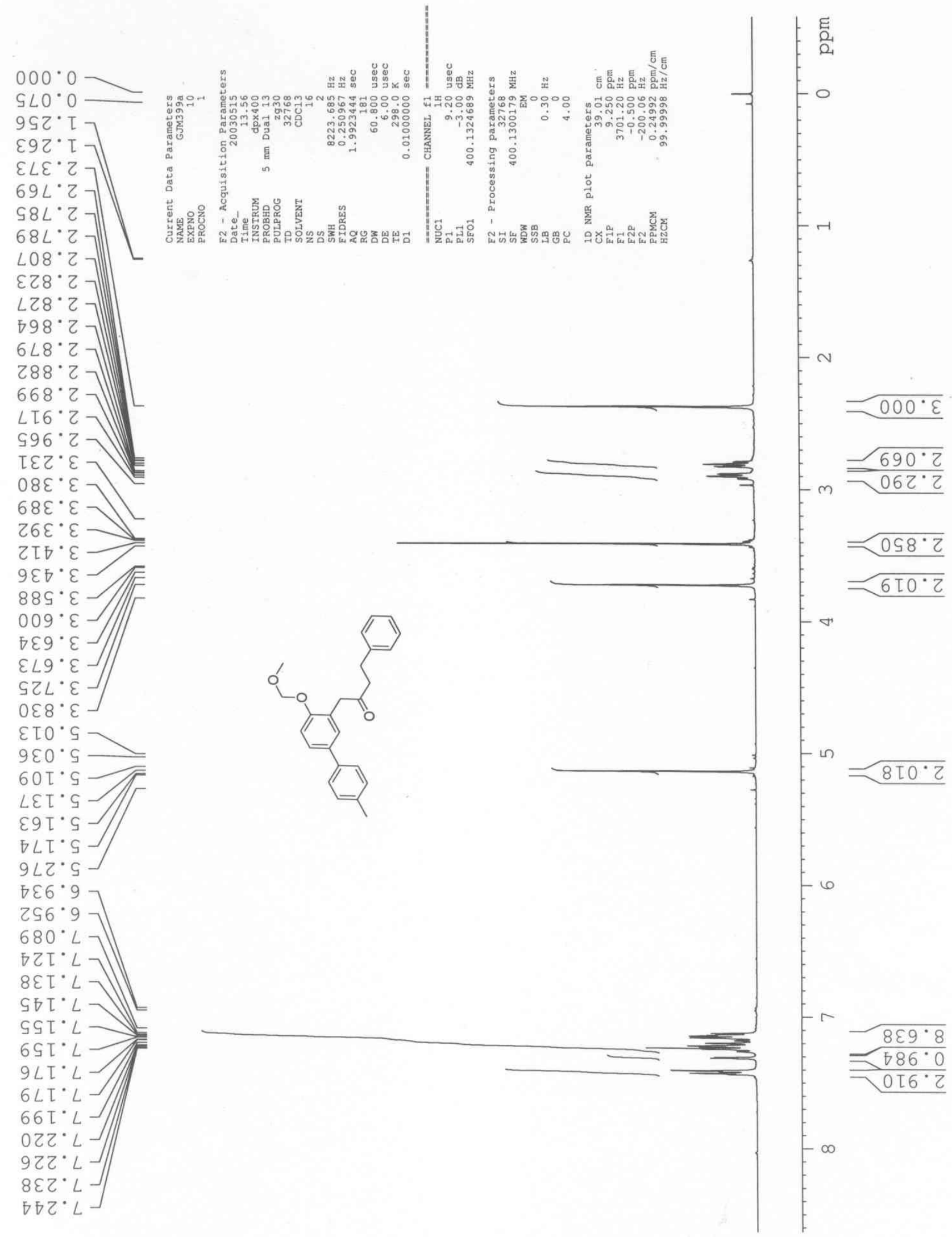


1-[2'-Methoxymethoxy-5'-(4'--methoxyphenyl)phenyl]-4-phenylbutan-2-one 32ab'

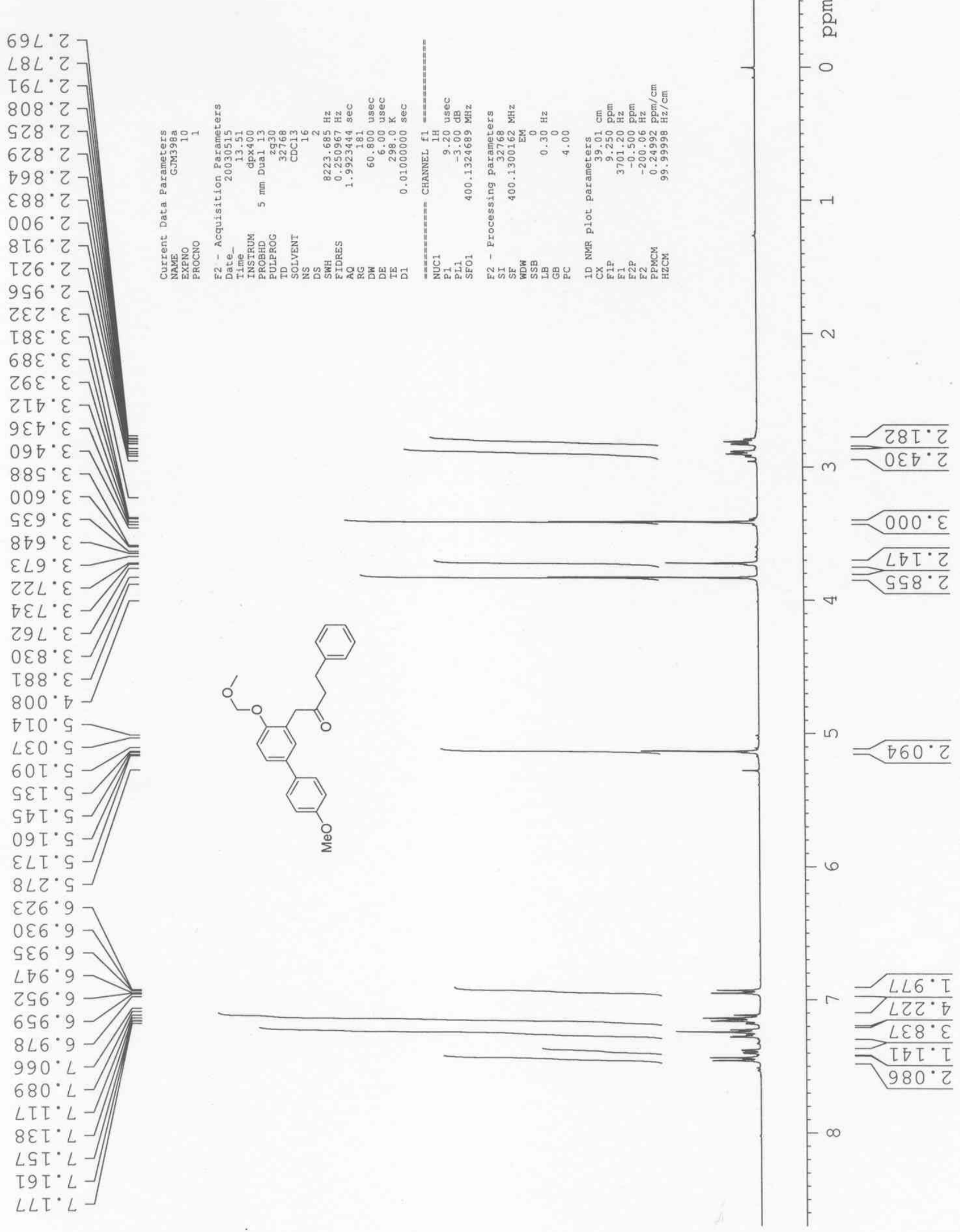


1-[2'-Methoxymethoxy-5'-(4"-nitrophenyl)phenyl]-4-phenylbutan-2-one 32ac'

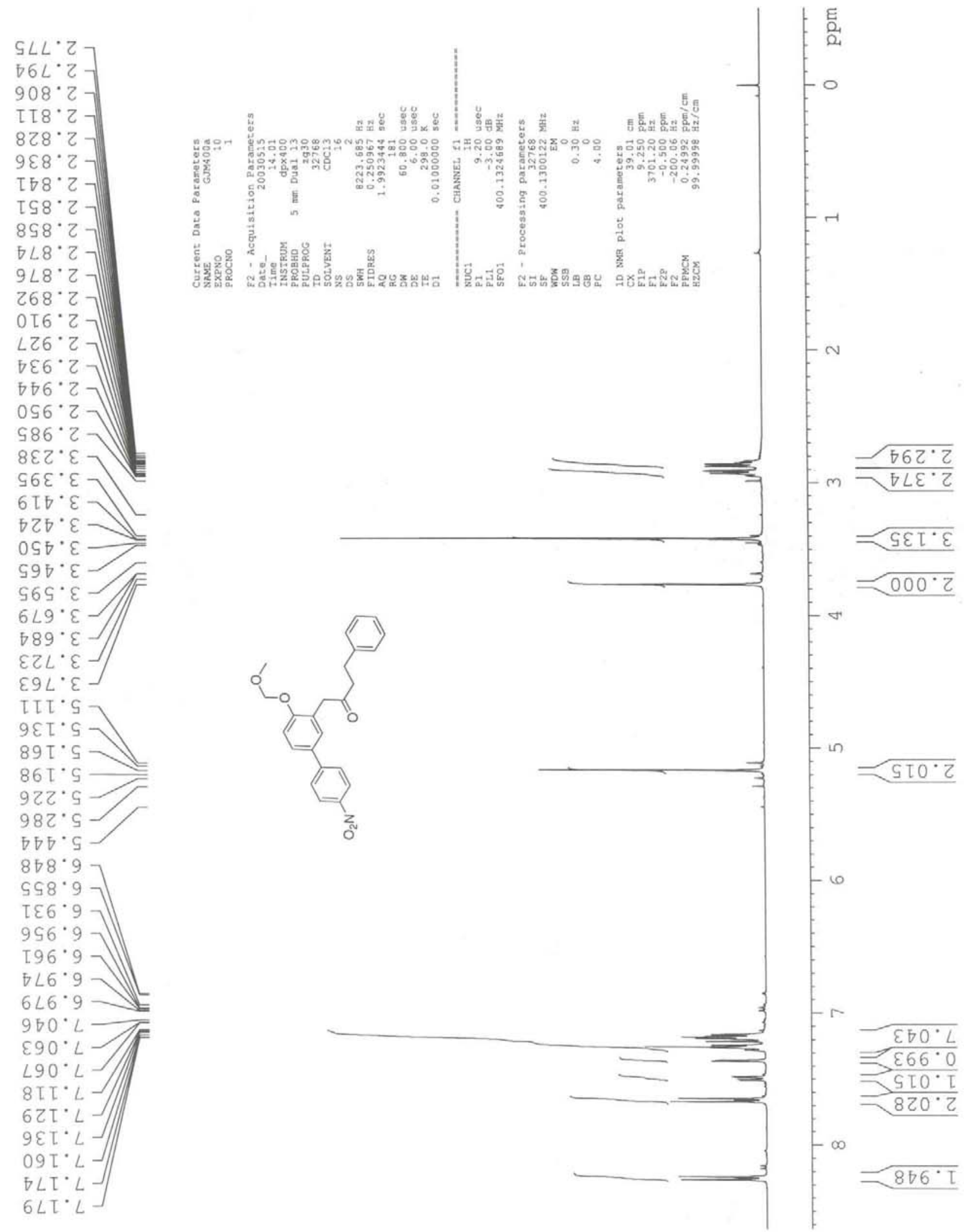


1-[2'-Methoxymethoxy-5'-(4"-methylphenyl)phenyl]-4,4-dimethylacrylone 32 $\mathrm{ba}^{\prime}$

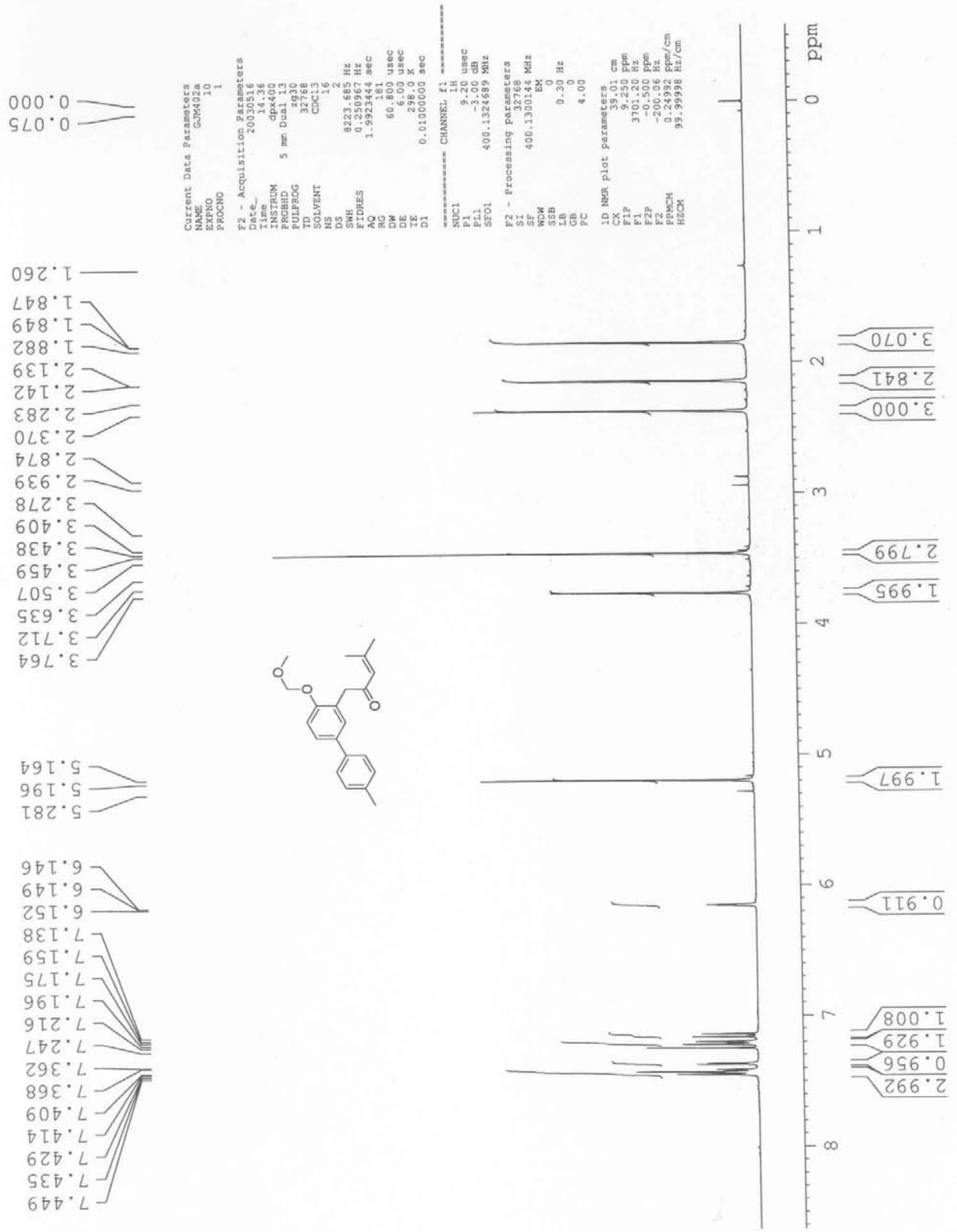


1-[2'-Methoxymethoxy-5'-(4"-methoxyphenyl)phenyl]-4,4-dimethylacrylone 32bb'
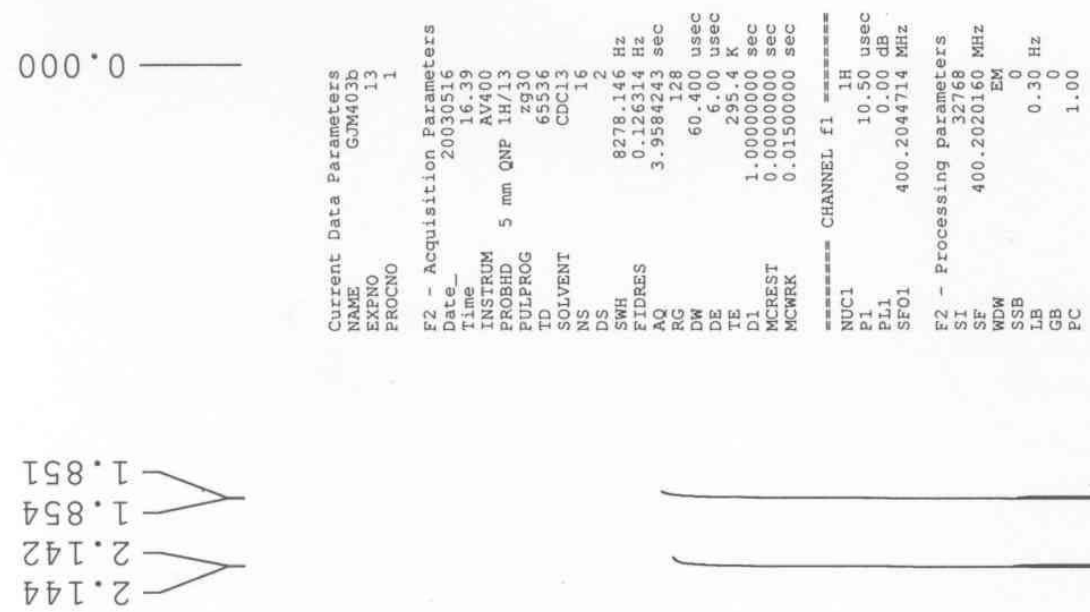

$69 \nabla^{\circ} \cdot \mathcal{-}$

$\nabla 9 L^{\circ} \mathcal{E}$

โE $8 \cdot \varepsilon \longrightarrow$
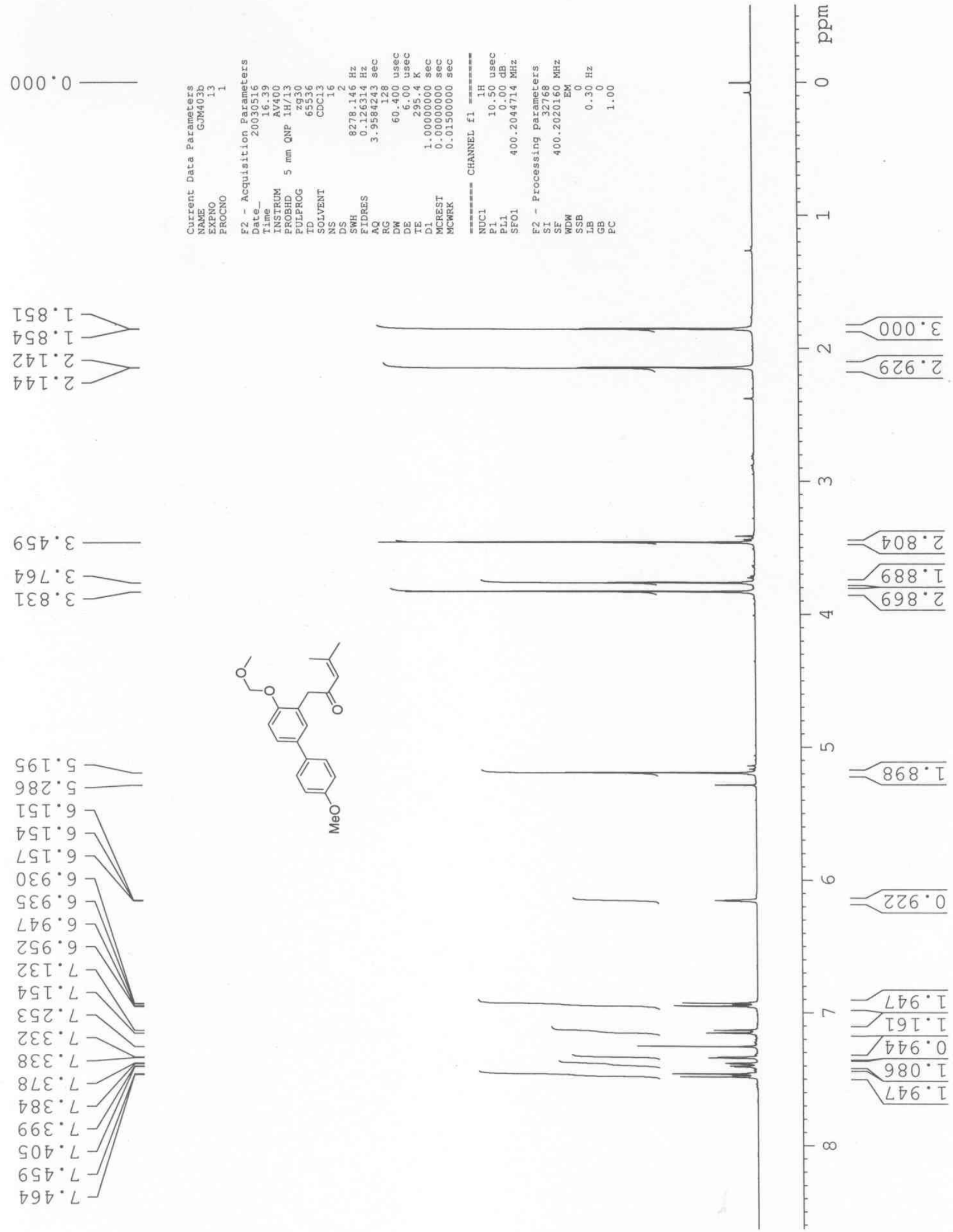
1-[2'-Methoxymethoxy-5'-(4"-nitrophenyl)phenyl]-4,4-dimethylacrylone 32bc'

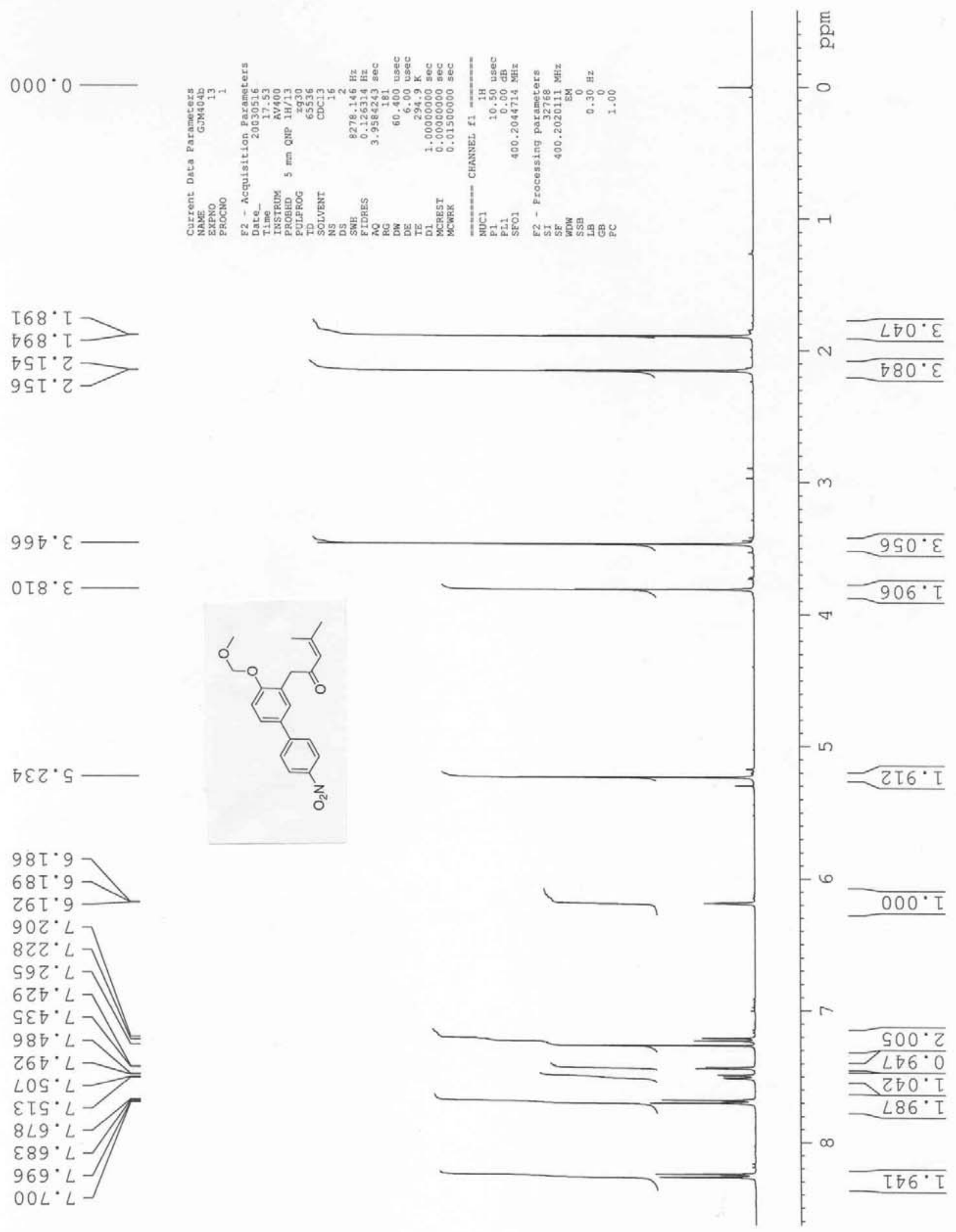



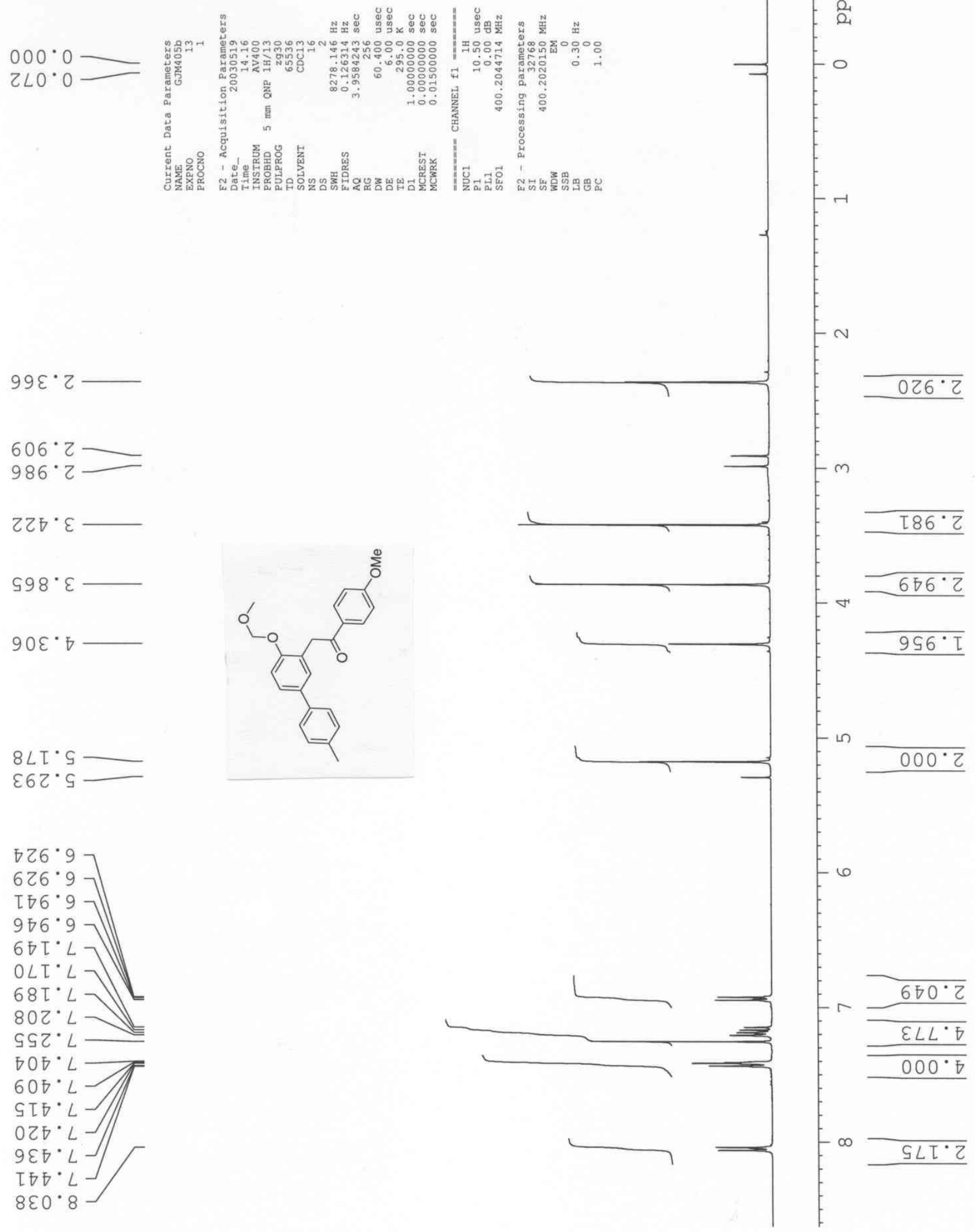


\section{1-[2'-Methoxymethoxy-5'-(4"'-methoxyphenyl)phenyl]-para-methoxyacetophenone 32cb'}

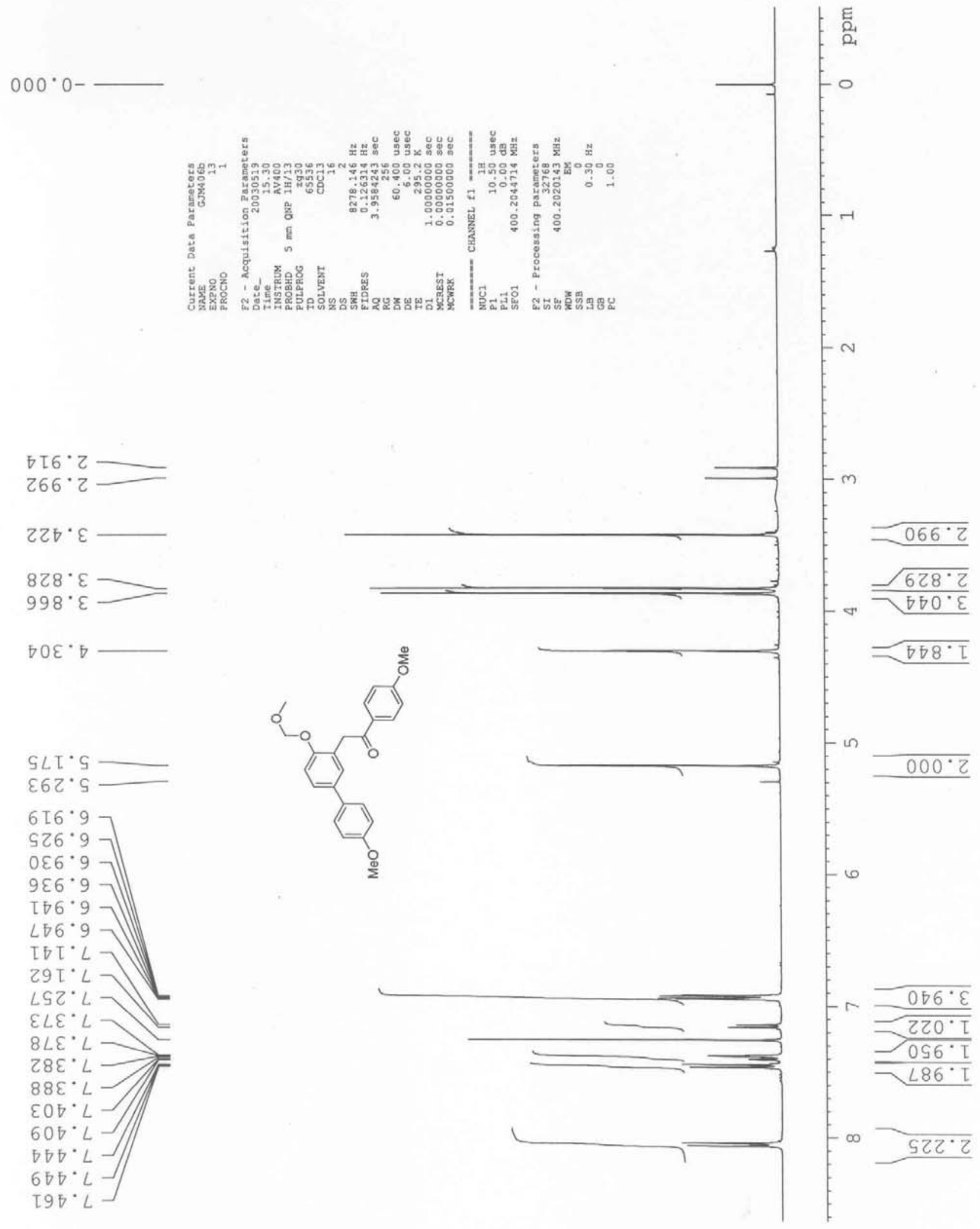




\section{1-[2'-Methoxymethoxy-5'-(4"-nitrophenyl)phenyl]-para-methoxyacetophenone 32cc'}

$000^{\circ} 0-$
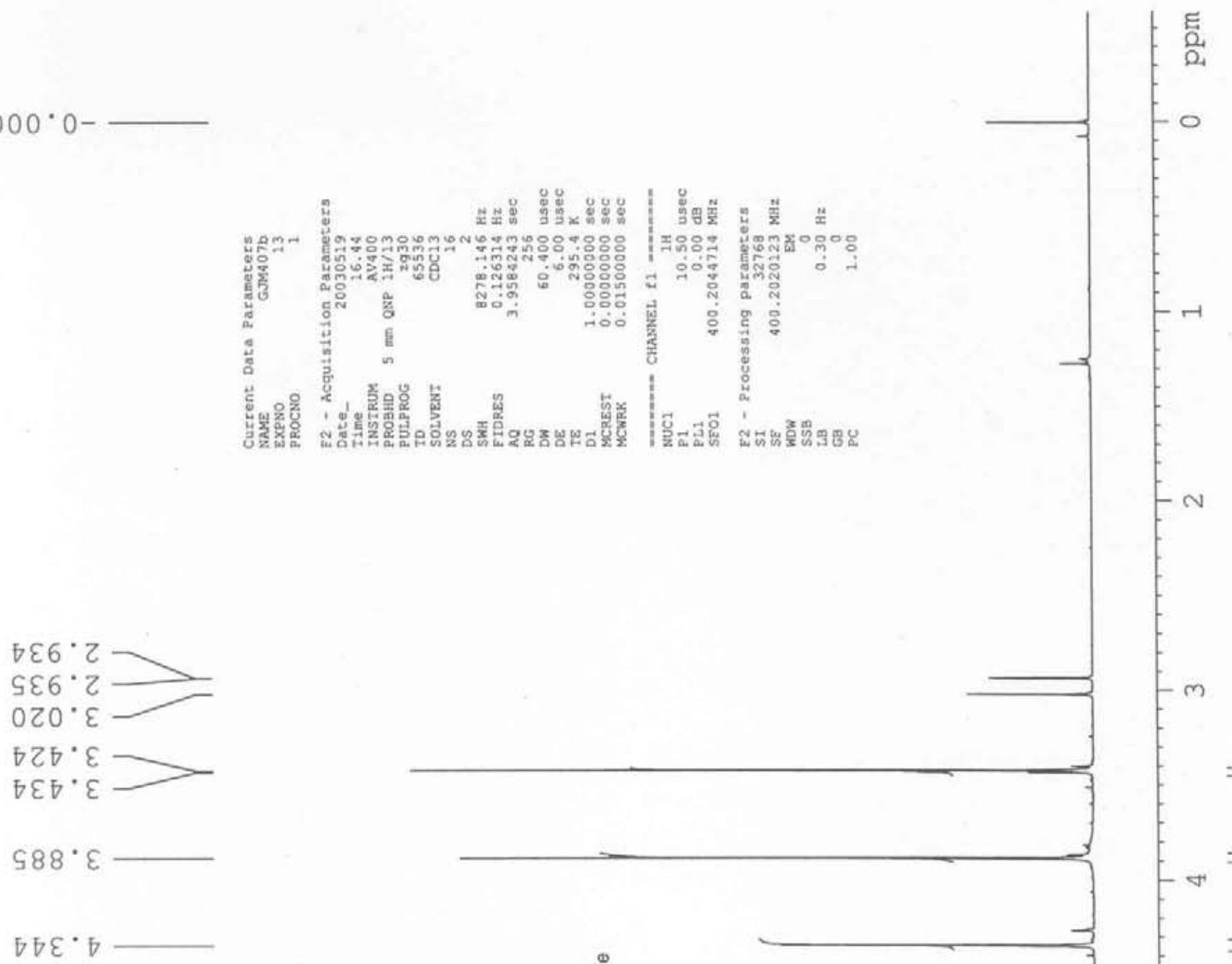

$802^{\circ} 9$
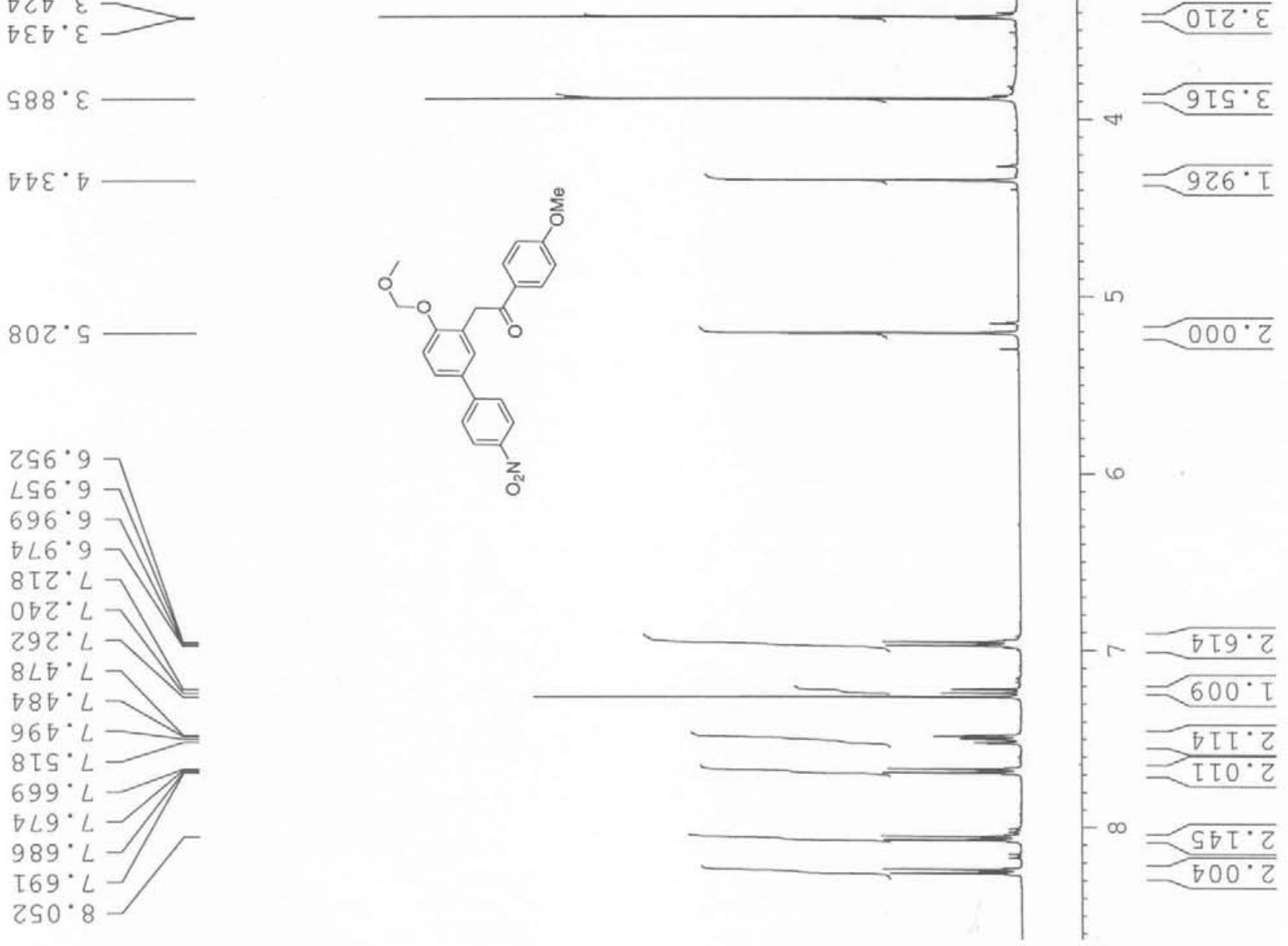
${ }^{1} \mathrm{H}$ NMR spectra of benzo[b]furans 33aa'-cc'

\section{5-(4"-Methylphenyl)-2-(2'-phenylethyl)benzo $[b]$ furan 33aa'}
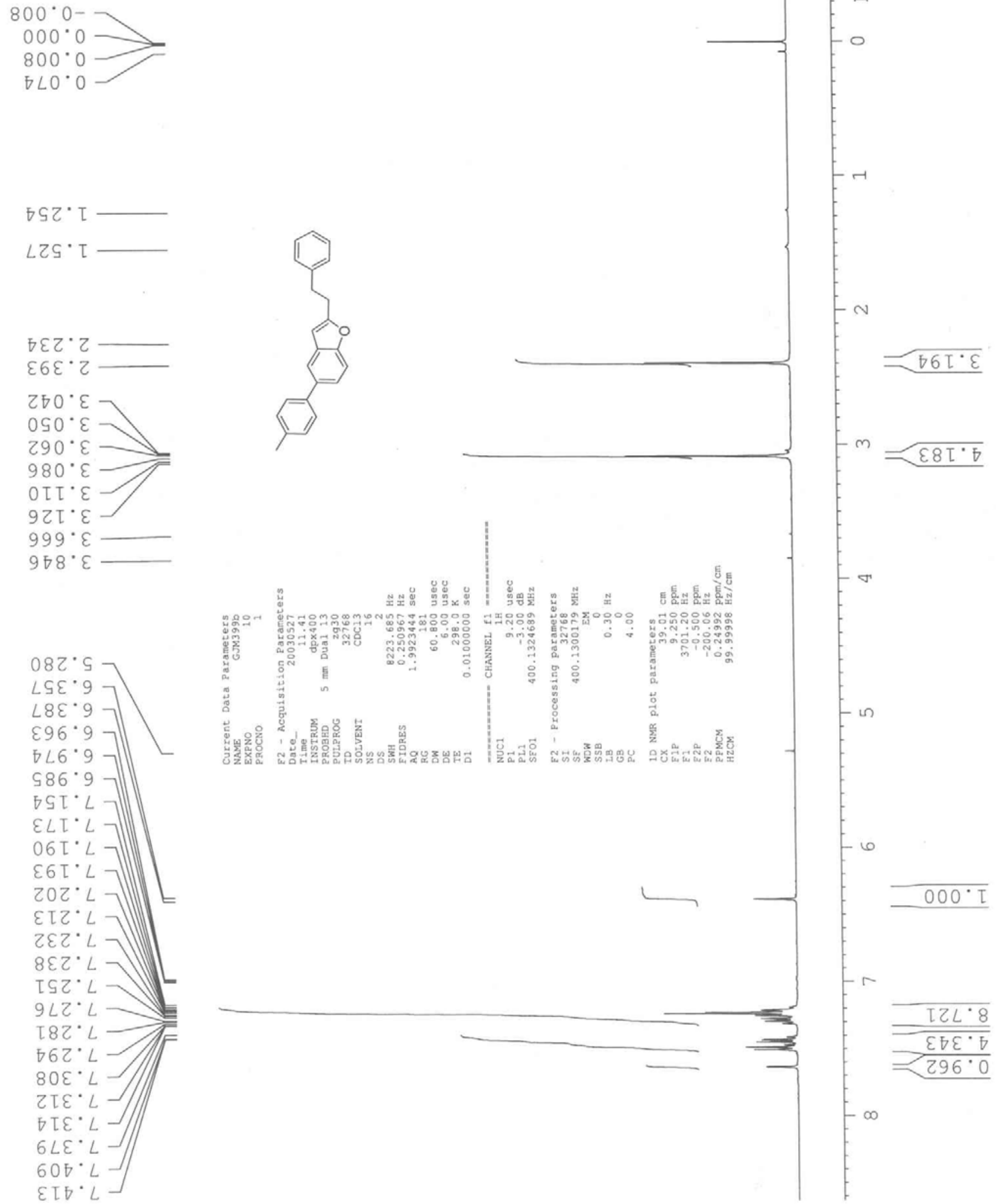
5-(4"'-Methoxyphenyl)-2-(2'-phenylethyl)benzo[b]furan 33ab'
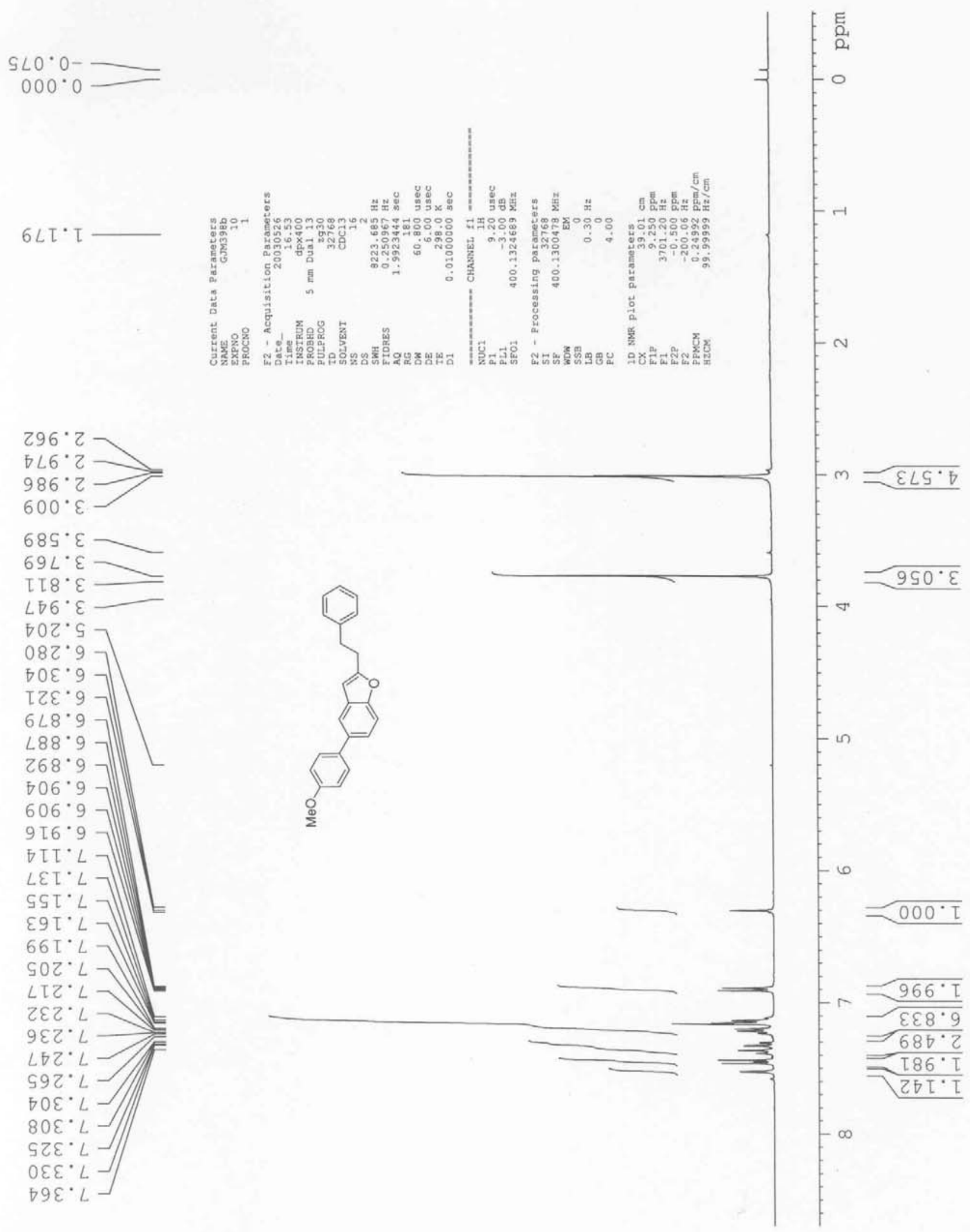


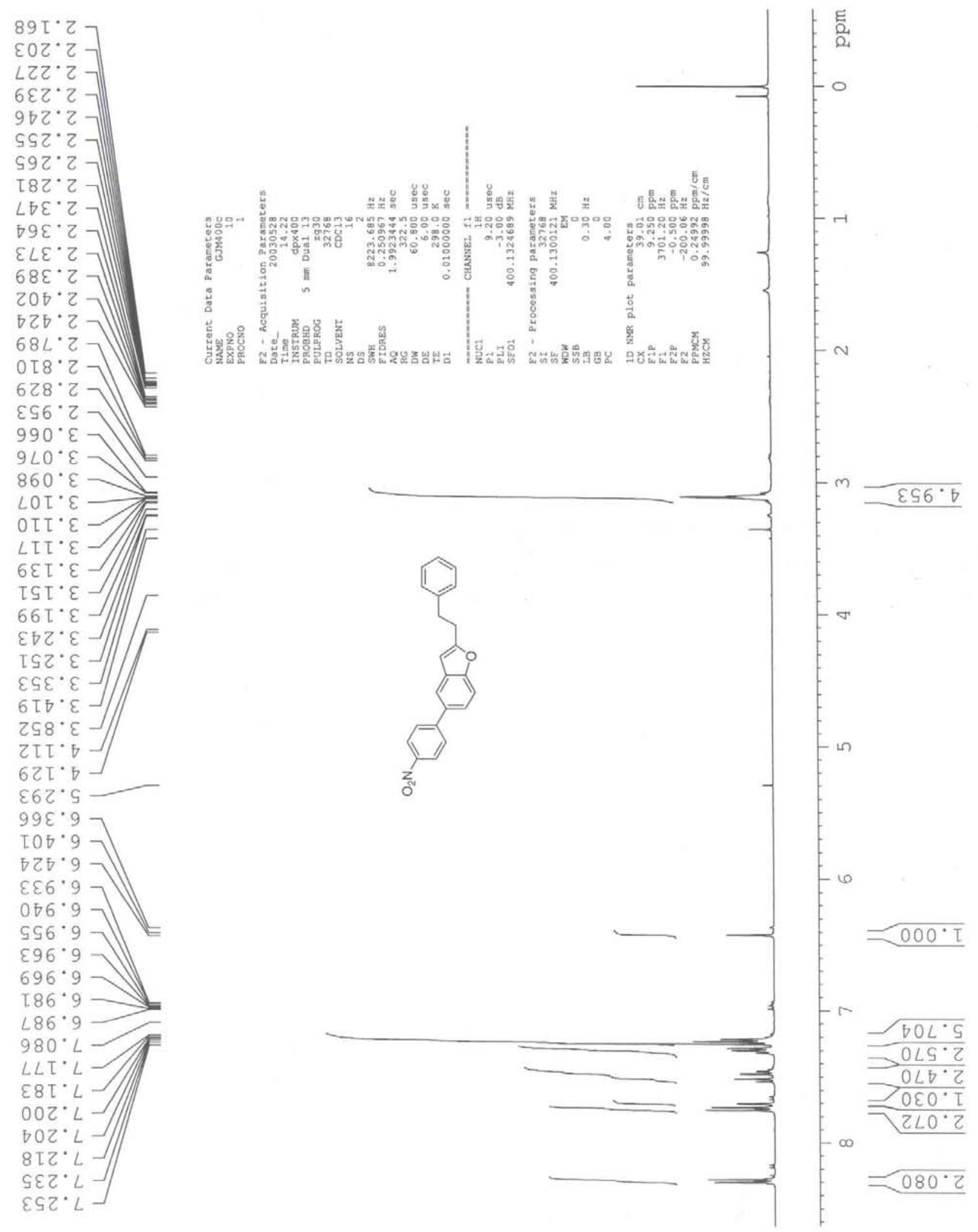


5-(4'-Methylphenyl)-2-(2',2'-dimethylethenyl)benzo[b]furan 33ba'

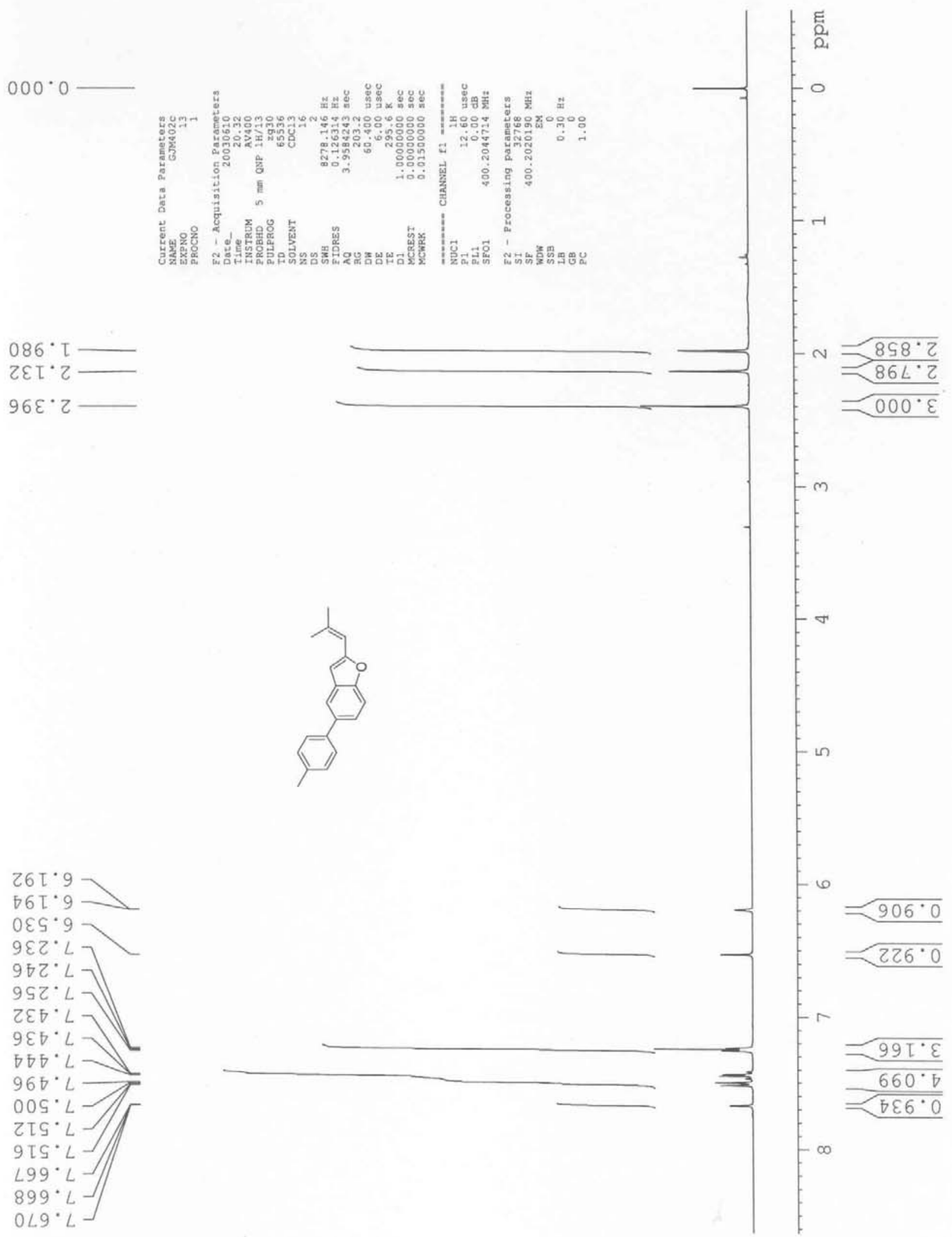




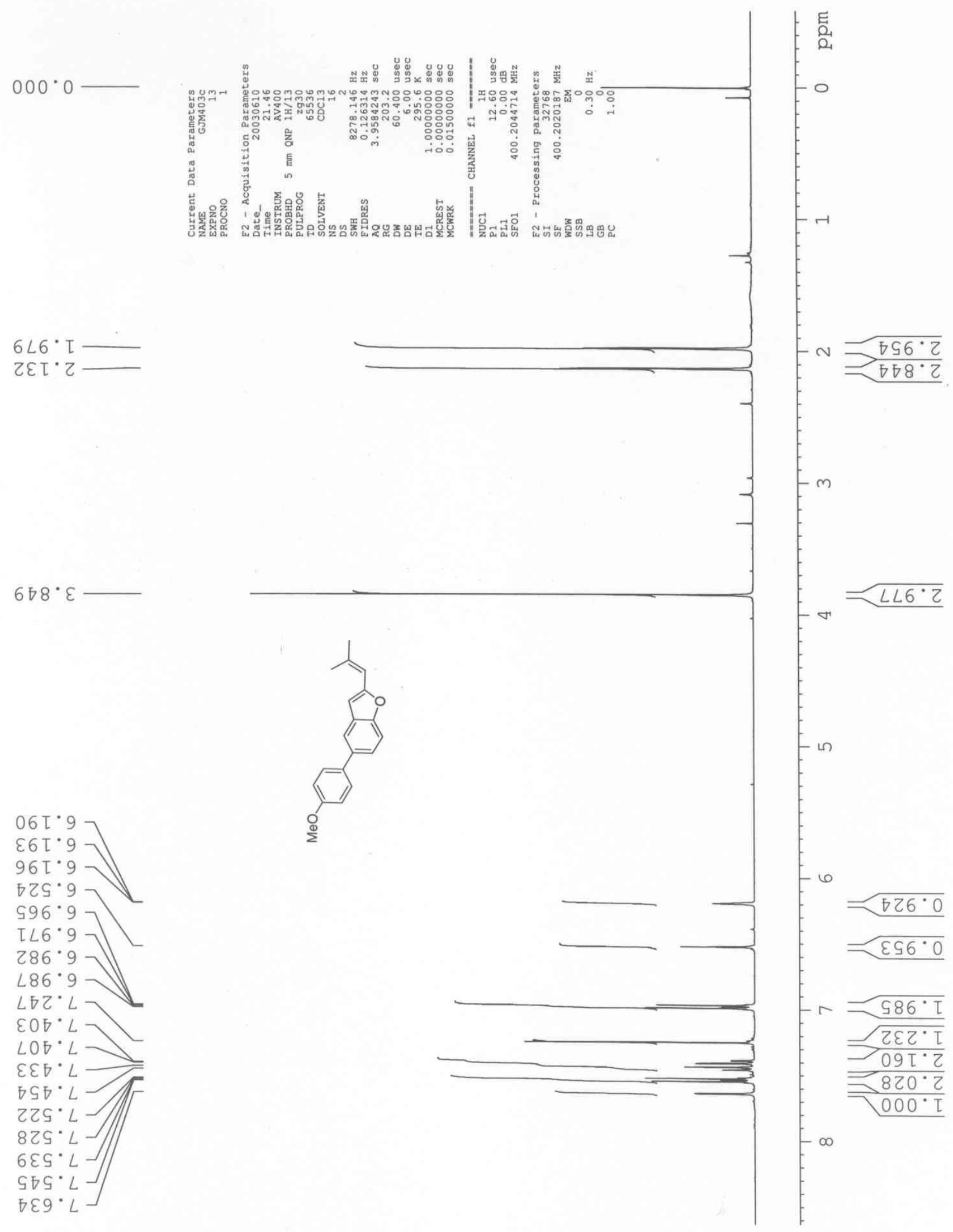


5-(4"-Nitrophenyl)-2-(2',2'-dimethylethenyl)benzo[b]furan 33bc'

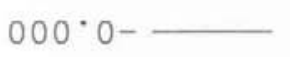

$866^{\circ} \mathrm{T}$ ZE T' $\mathrm{Z}$ $9 \nabla T \cdot z$

$\mathrm{S}^{\circ} \mathrm{C}^{\cdot} \mathrm{\tau}$

$\nabla \tau \varepsilon^{*} \varepsilon$

$96 Z^{\circ} \mathrm{G}$

$\nabla 0 Z \cdot 9$

$\mathrm{LOZ} \cdot 9$

0 T $2 \cdot 9$

LSS. 9

$\angle G Z^{\circ}$

OSE ${ }^{\circ} L$

$\nabla S \sigma^{\circ} L$

TLD

$9 \angle \nabla^{\circ} L$

EOS ${ }^{\circ} L$

$\nabla Z S^{\circ} L$

IEL ${ }^{*} L$

$S E L^{\circ} L$

$O B L^{\circ} L$

ZGL ${ }^{\circ} L$

$\angle S L^{\circ} L$

$\varepsilon 9 L^{\circ} L$
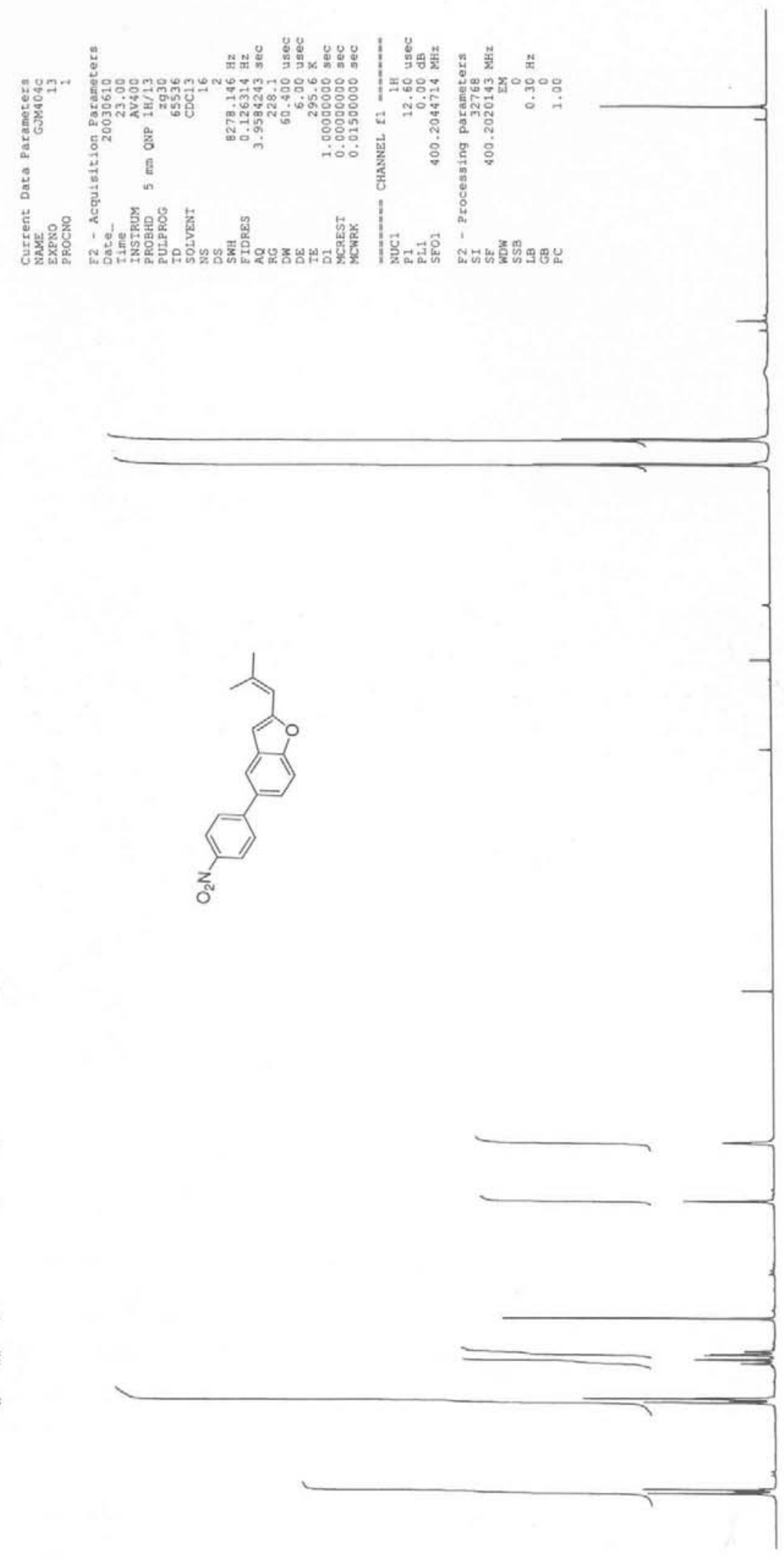

틈

ڤ

o

N

$\frac{760^{\circ} \varepsilon}{950^{\circ} \varepsilon}$

m

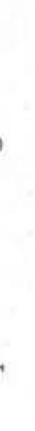

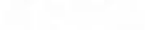


5-(4"-Methylphenyl)-2-(4'-methoxyphenyl)benzo[b]furan 33ca'

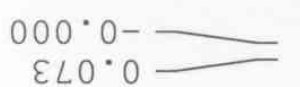

$500^{\circ} 2$

T98 $\mathcal{~} \mathcal{E}$

$\angle 06 \cdot 9$

$606^{\circ} 9$

IL $6^{\circ} 9$

$9 L 6 \cdot 9$

$886 \cdot 9$

$\varepsilon 66^{\circ} 9$

$8 \nabla \tau^{*} L$

$992^{\circ} L$

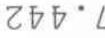

$\angle \nabla 0^{\circ} L$

$790^{\circ} \mathrm{L}$

$890^{\circ} \mathrm{L}$

$\varepsilon \tau S^{*} L$

$\varepsilon \varepsilon S^{*} L$

โโL $\iota^{*} L$

SIL $L^{\circ} L$

$S 6 L^{\circ} L$
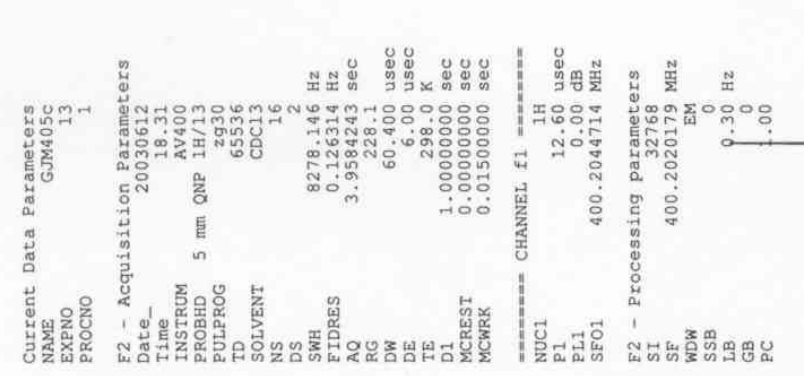

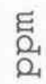

$-0$<smiles>Cc1ccc(-c2ccc3oc(-c4ccc([O])cc4)cc3c2)cc1</smiles>

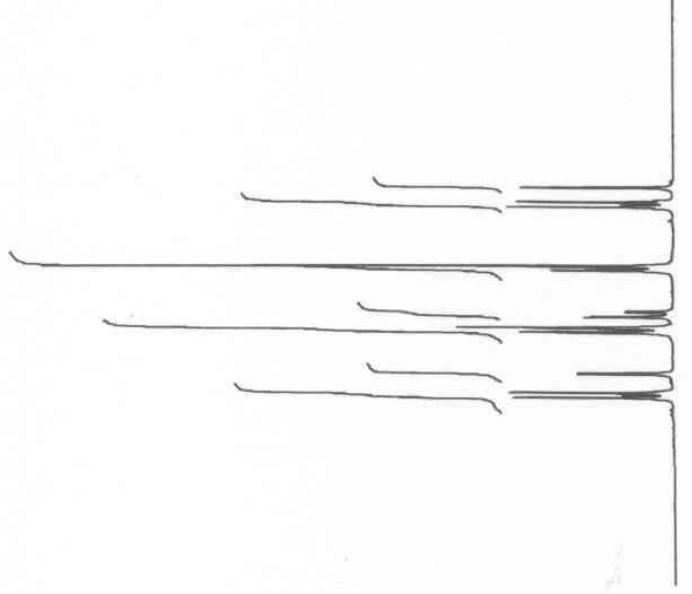

$=$

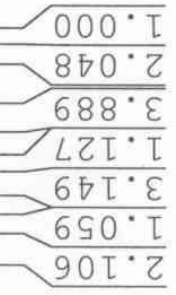




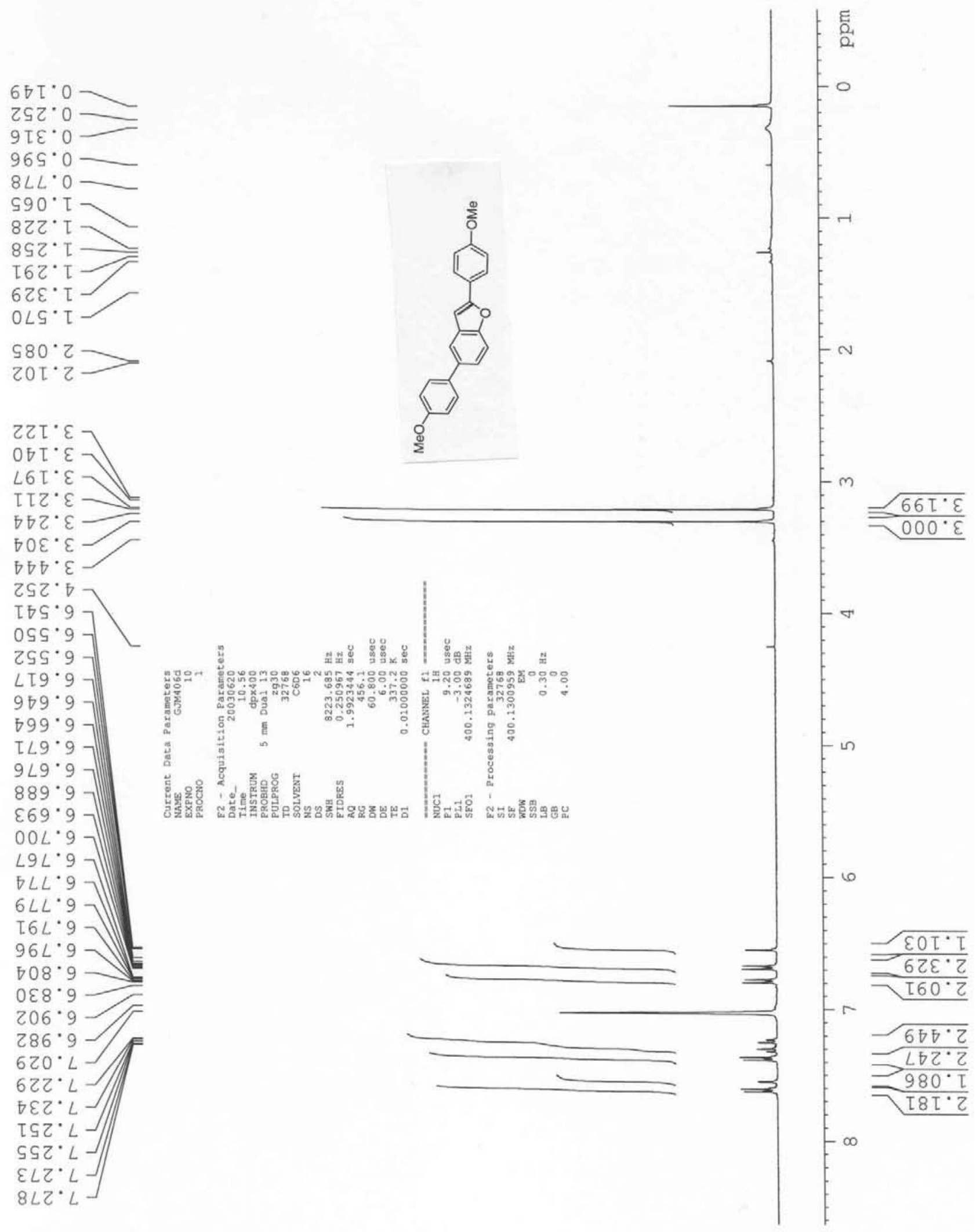



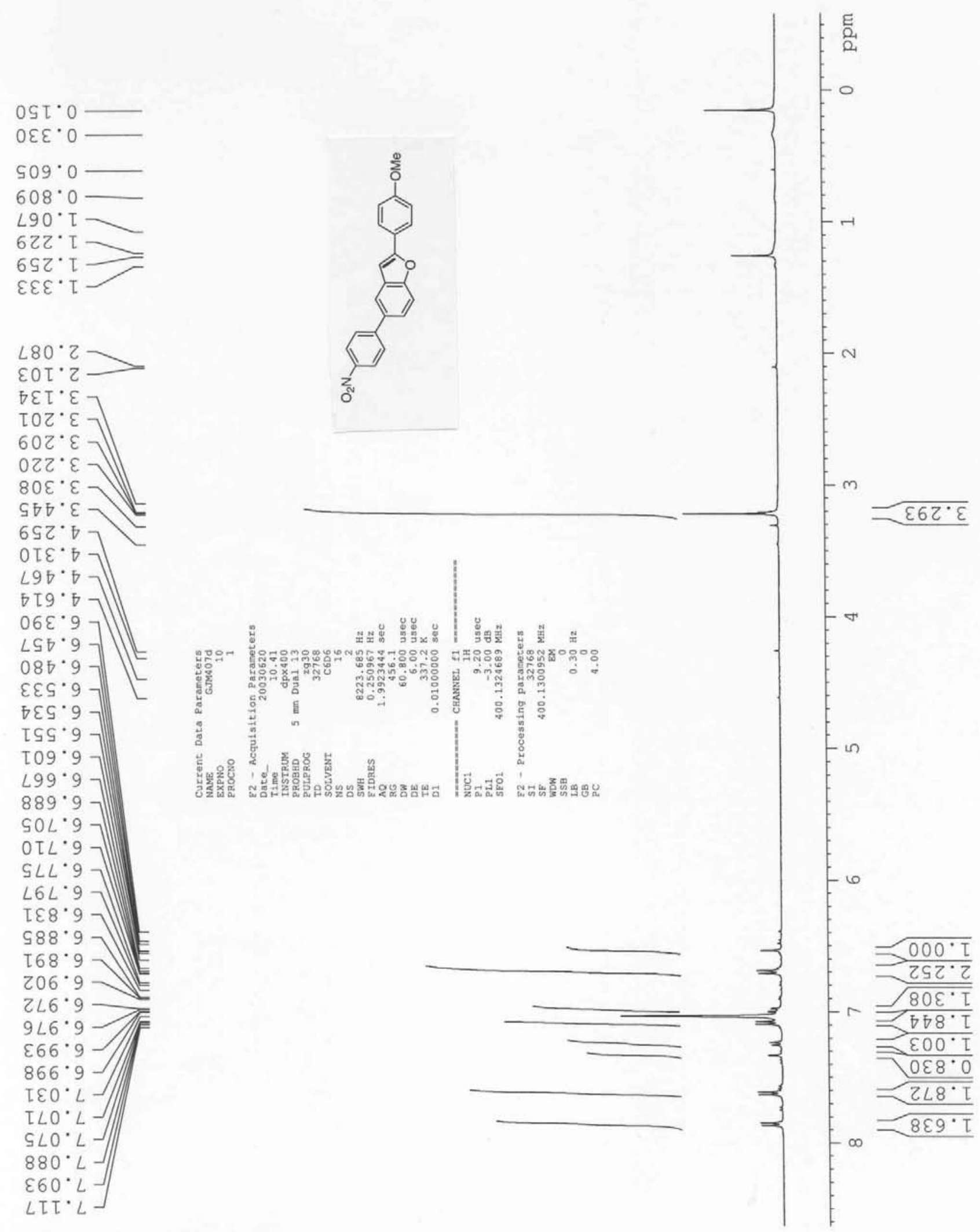